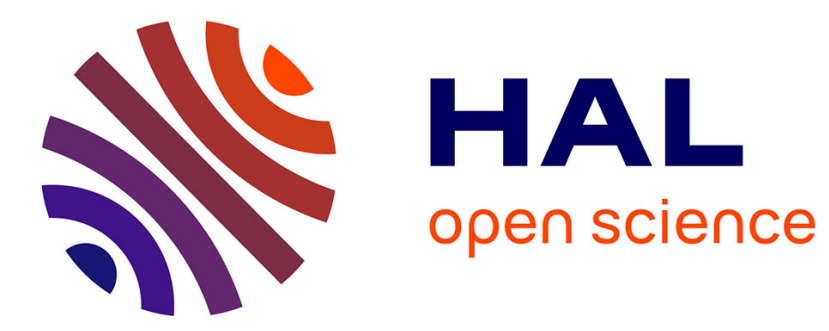

\title{
4D Anisotropic Diffusion Framework with PDEs for Light Field Regularization and Inverse Problems
}

Pierre Allain, Laurent Guillo, Christine Guillemot

\section{To cite this version:}

Pierre Allain, Laurent Guillo, Christine Guillemot. 4D Anisotropic Diffusion Framework with PDEs for Light Field Regularization and Inverse Problems. IEEE Transactions on Computational Imaging, 2019, pp.1-16. 10.1109/TCI.2019.2919229 . hal-02135541

\section{HAL Id: hal-02135541 \\ https://hal.science/hal-02135541}

Submitted on 21 May 2019

HAL is a multi-disciplinary open access archive for the deposit and dissemination of scientific research documents, whether they are published or not. The documents may come from teaching and research institutions in France or abroad, or from public or private research centers.
L'archive ouverte pluridisciplinaire HAL, est destinée au dépôt et à la diffusion de documents scientifiques de niveau recherche, publiés ou non, émanant des établissements d'enseignement et de recherche français ou étrangers, des laboratoires publics ou privés. 


\title{
4D Anisotropic Diffusion Framework with PDEs for Light Field Regularization and Inverse Problems
}

\author{
Pierre Allain, Laurent Guillo, Christine Guillemot, Fellow, IEEE \\ Inria Rennes Bretagne-Atlantique, 263 Avenue Général Leclerc, 35042 Rennes
}

\begin{abstract}
In this paper, we consider the problem of vectorvalued regularization of light fields based on PDEs. We propose a regularization method operating in the 4D ray space that does not require prior estimation of disparity maps. The method performs a PDE-based anisotropic diffusion along directions defined by local structures in the 4D ray space. We analyze light field regularization in the $4 \mathrm{D}$ ray space using the proposed $4 \mathrm{D}$ anisotropic diffusion framework by first considering a light field toy example, i.e., a tesseract. This simple light field example allows an in-depth analysis of how each eigenvector influences the diffusion process. We then illustrate the diffusion effect for several light field processing applications: denoising, angular and spatial interpolation, regularization for enhancing disparity estimation as well as inpainting.
\end{abstract}

Index Terms-Light Field, regularization, anisotropic diffusion, inverse problems

\section{INTRODUCTION}

$\mathbf{I}$ NVERSE problems appear in a plethora of image processing and computer vision applications. Many image processing problems can indeed be formalized as the recovery of an image given noisy or incomplete observations. This is for example the case of image denoising, inpainting or super-resolution. A common strategy for dealing with ill-posed inverse problems consists in introducing some prior knowledge on the kind of typical images we try to restore, which helps restricting the class of admissible solutions. The smoothness prior has been one of the most widely used assumptions in computer vision together with regularization schemes using a variational regularization or partial differential equations (PDEs). To preserve edges in images, the regularization is often performed with some anisotropy, i.e. along privileged directions defined by structure tensors giving information on local image structures. These regularization schemes turned out to be very powerful tools for solving a number of inverse problems in $2 \mathrm{D}$ imaging.

As the capture of $4 \mathrm{D}$ light fields from real scenes grows in popularity, the need for solving similar inverse problems with these $4 \mathrm{D}$ data is expected to rise. However, the very large volume of data with angular consistency that needs to be preserved along the processing chain makes the above problems quite challenging. The authors in [1] propose a regularization scheme, leveraging the variational structure in light fields, for denoising and super-resolution. They use 2D structure tensors computed in epipolar plane images (EPI) to estimate disparity between light field views. With the help

This work was supported by the EU H2020 Research and Innovation Programme under grant agreement No 694122 (ERC advanced grant CLIM) of the estimated disparity, they project all the views on a target viewpoint and solve the regularization using a global optimization framework with total variation (TV) priors. The final regularizer is written as the contributions of regularizers on vector fields in EPI 2D spaces, with user-defined constants which adjust the amount of smoothing on the different views or EPIs. Errors in disparity maps may however translate into annoying artefacts in particular along edges.

In this paper, we propose instead a regularization method operating directly in the 4D ray space that does not require prior estimation of disparity maps. The method performs a PDE-based diffusion with anisotropy steered by a tensor field based on local structures in the 4D ray space that we extract using a 4D tensor structure. To enhance coherent structures, the smoothing along directions, surfaces, or volumes in the $4 \mathrm{D}$ ray space is performed along the eigenvectors directions. Although anisotropic diffusion is well understood for 2D imaging, its interpretation and understanding in the 4D space is far from being straightforward. We first analyse the behaviour of the diffusion process on a light field toy example, i.e. a tesseract (a 4D cube). We particularly illustrate the effect of the diffusion along each of the 4 eigenvectors forming the diffusion tensors.

The proposed ray space regularizer is a tool enabling us to tackle a variety of inverse problems in the ray space. We first illustrate the potential of the proposed 4D anisotropic diffusion for light field denoising. This led us to introduce a novel denoising method that we called Anisotropic Diffusion Denoising 4D (ADD4D). Denoising results show that the proposed method compares well to the best state of the art method [2], even outperforming it at high noise levels, while being considerably faster. Preliminary light field denoising results with ADD4D have been presented in [3]. However, in this paper, in addition to the in-depth analysis on the tesseract toy example, we further revise the tuning of the model parameters aiming at a more intuitive use. We then demonstrate the ability of the presented approach to perform both angular and spatial light field interpolation. The use of the proposed anisotropic diffusion framework as a 4D regularization of the light field, by removing inter-view inconsistencies and noise, is also shown to improve disparity estimation in epipolar plane images. We finally illustrate the effect of the proposed framework in the context of inpainting.

\section{RELATED WORK}

In this section, we give a brief review of methods related to our work, starting with the main regularization and diffusion 
methods widely used in 2D image processing. We then review the few papers of the litterature dealing with regularization for inverse problems in light field processing.

2D regularization. The goal here is not to give a complete review of the numerous methods for $2 \mathrm{D}$ regularization, but instead to briefly remind the principles of those related to this work. This section therefore focuses on PDE based regularization. The framework of diffusion based on PDEs has been widely used for image regularization, going from classical isotropic diffusion, to non linear diffusion [4] and anisotropic smoothing [5]. Although sometimes referred to as anisotropic diffusion, non linear diffusion locally adapts the response of the smoothing filter so that the diffusion process is lessened near edges and is stronger in homogenous areas. One step further has been to introduce locally adapted filters for performing truly anisotropic diffusion close to image structures such as edges [5]. Anisotropic regularization (or diffusion) refers to a smoothing in privileged spatial directions with different weights. The author in [5] uses a field of diffusion tensors to find privileged directions of diffusion. The diffusion tensor $\mathbf{T}$ (symmetric and positive-definite matrix) is derived in each point $\mathbf{p}=(x, y)$ of the image from the spectral elements of the structure tensor as:

$$
\mathbf{T}=\lambda_{+} \theta_{+} \theta_{+}^{T}+\lambda_{-} \theta_{-} \theta_{-}^{T},
$$

where $\theta_{-}$and $\theta_{+}$are the eigenvectors, and $\lambda_{-}$and $\lambda_{+}$the corresponding eigenvalues, of the $2 \times 2$ symmetric and semipositive-definite matrix called structure tensor (also called the Di Zenzo tensor [6]) defined as $T=\nabla I \nabla I^{T}$. Note also that the tensor can be regularized with Gaussian kernels, in order to have increased coherency in the retrieved geometry.

A number of anisotropic diffusion methods have then been proposed, whose main differences lie first on the definition of the diffusion tensor defining the smoothing geometry, and on the PDE used to perform the smoothing. To give an example, coherence-enhancing anisotropic diffusion is proposed in [7], which increases the diffusivity along the direction given by $\theta_{-}$when the coherence measured by $\left(\lambda_{+}-\lambda_{-}\right)^{2}$ increases. The PDE performing the smoothing can also take several forms, using a divergence-based formulation [5], a trace-based formulation [8]. In [9], [10], the smoothing is modelled as the juxtaposition of two oriented 1D Gaussian smoothing processes along orthonormal directions with different weights. The link between these three formulations is established in [8]. The author in [11] further introduces a curvature-preserving PDE that aims at respecting specific curvature constraints, by adding to the trace-based PDE a term taking curvatures of integral curves of the diffusion tensor field into account. A graph Laplacian regularizer is proposed in [12] for 2D image denoising, assuming that the original image (patch) is smooth with respect to a defined graph in which vertices correspond to pixels on a $2 \mathrm{D}$ grid connected via edges. However, with high dimensional data as light fields, the dimension of the graph can rapidly become an issue.

Light field inverse problems and regularization. Although, at an early stage, inverse problems in light field imaging have naturally arised with the emergence of real light field cameras. Research effort has in particular been dedicated to denoising and super-resolution, in particular for overcoming technological limitations of plenoptic cameras. The authors in [13] use a Gaussian mixture model (GMM) prior for light field patches, and then exploit this prior within a Bayesian inference framework for light field denoising. In [14], the light field is denoised by stacking EPIs in a 3D volume using the VBM4D video denoising method [15], while the authors in [16] apply a hyper-fan shaped filter in a 4D discrete Fourier transform domain. The BM3D denoising algorithm [17] is extended in [2] to light fields. The only approach of regularization proposed so far for light field denoising is the one of [1] using a global variational framework integrating contributions of regularizers on vector fields computed in EPI 2D spaces. Regularization based on anisotropic total variation is also used in [18] for light field deblurring. A regularization term is used to enforce the equiparallax constraint, hence to enforce valid light field geometry.

Light field spatial and angular super-resolution has also received a lot of attention [19], [20], [13], [21] using either Gaussian priors in Bayesian inference frameworks or learning methods as in [21] and [22]. In a similar manner as in [1], the authors in [23] first estimate disparity maps for each view and project all the views into the target one within a global variational optimization framework. Errors in disparity maps may however translate into annoying artefacts in the superresolved light field.

Another important light field processing problem that can benefit from the proposed regularization framework is light field view interpolation. Existing approaches for view interpolation can be broadly divided into two main categories. A first category of methods relying on depth image based rendering techniques makes explicit use of geometric or depth information to warp source views onto target viewpoints. The various existing methods differ in the way scene geometry is estimated, e.g. using optical flows between the input views as in [24] or using structure tensors computed in epipolar plane images as in [25] where the authors cast the view synthesis problem in a variational framework. However, the quality of the synthesized views obtained by the above approaches in general very much depend on the quality of the depth or disparity information which is difficult to estimate in textureless and occluded regions. The quality of depth maps can also be enhanced thanks to anisotropic regularization as in [26] where the authors proposed a method based on active wave-font sampling and the variational principle to regularize the depth map of the center view.

A second category of methods based on deep learning techniques have also recently appeared either explicitly or implicitly exploiting depth information. An architecture is proposed in [27] based on first deep convolutional neural network (CNN) to perform spatial super-resolution followed by a second CNN to synthesize intermediate views. The authors in [28] use a convolutional neural network to estimate novel views from a set of input views with wide baseline. An architecture based on a cascade of two convolutional neural networks is proposed in [29] to estimate both the disparity maps and color of synthesized views from the four corner views of a dense light field with small disparity. 
In this paper, we propose instead a regularization scheme directly operating in the $4 \mathrm{D}$ ray space that can be applied to solve inverse problems in light field processing. The method does not require prior depth estimation. We first demonstrate the interest of the proposed approach for three applications: denoising, angular super-resolution or view interpolation, and spatial interpolation. We further illustrate the potential for light field inpainting as well as for regularizing light fields in order to improve the quality of estimated depth maps.

\section{4D ANISOTROPIC DIFFUSION}

Let $L(x, y, u, v)$ denote the $4 \mathrm{D}$ representation of a light field, describing the radiance of a light ray parameterized by its intersection with two parallel planes [30], and where $(u, v)$ denote the angular (view) coordinates and $(x, y)$ the spatial (pixel) coordinates.

\section{A. $4 D$ structure tensor}

To perform light field regularization along pre-defined directions which would preserve edges, one has to first characterize local structures in the image, or here the light field. While light field regularization methods are usually applied per Epipolar Plane Image (EPI) of the light field with directions given by $2 \mathrm{D}$ structure tensors often smoothed with a $2 \mathrm{D}$ Gaussian kernel, we consider instead a direct computation of 4D structure tensors in the 4D ray space, which is defined as the symmetric and semipositive definite $4 \times 4$ matrix [31]:

$$
\mathbf{T}=\frac{1}{C} \sum_{c=1}^{C} \nabla L_{c} \otimes \nabla L_{c}^{\top},
$$

where $C$ is the number of color channels. The notation $\nabla L_{c}$ denotes the gradient or partial derivatives in the 4 dimensions of the color channel $L_{c}$. A smoothed version $\mathbf{T}_{\omega}=\mathbf{T} *$ $G_{\omega}(x, y, u, v)$ of the structure tensor is in practice computed to retrieve a more coherent geometry, where $G_{\omega}(x, y, u, v)$ is a Gaussian kernel smoothing along the four light field dimensions, with $\omega=\left[\omega_{x}, \omega_{y}, \omega_{u}, \omega_{v}\right]$. Its spectral elements, i.e. the eigenvectors $\nu_{i}, i=1 \ldots 4$ and the eigenvalues $\lambda_{i}, i=1 \ldots 4$ of the 4D structure tensor $\mathbf{T}$ respectively give the orientations of the local structures in the 4D ray space and the vectorvalued variations along these structures. We therefore have:

$$
\mathbf{T}_{\omega}=\sum_{i=1}^{4} \lambda_{i} \nu_{i} \nu_{i}^{T}
$$

The eigenvector $\nu_{1}$ with the largest eigenvalue $\lambda_{1}$ gives the dominant orientation of the local structures in the 4D ray space. The ratios between the eigenvalues gives information on the type of structure present in the local neighborhood.

\section{B. Trace-based PDE Diffusion}

To be adapted to the local structures in the 4D ray space of the light field, the diffusion process must be driven by a specific tensor, called diffusion tensor chosen so that it has the same set of eigenvectors $\nu_{i}, i=1 \ldots 4$ as the structure tensor T. A diffusion tensor is therefore constructed as:

$$
\mathbf{D}=\sum_{i=1}^{4} \beta_{i} \nu_{i} \nu_{i}^{T},
$$

where the coefficients $\beta_{i}$ set the smoothing strength along the direction given by $\nu_{i}$, and are chosen according to the goal of the diffusion.

The diffusion is thus formulated by the trace-based PDE:

$$
\frac{\partial L_{c}}{\partial t}=\operatorname{trace}\left(\mathbf{D H}_{c}\right) \text {, }
$$

where $\mathbf{H}_{\mathbf{c}}$ is the 4D Hessian matrix of the $c$ channel of $L$, defined as:

$$
\mathbf{H}_{\mathbf{c}}=\left(\begin{array}{llll}
\frac{\partial^{2} L_{c}}{\partial x^{2}} & \frac{\partial^{2} L_{c}}{\partial x \partial y} & \frac{\partial^{2} L_{c}}{\partial x \partial u} & \frac{\partial^{2} L_{c}}{\partial x \partial v} \\
\frac{\partial^{2} L_{c}}{\partial y \partial x} & \frac{\partial^{2} L_{c}}{\partial y^{2}} & \frac{\partial^{2} L_{c}}{\partial y \partial u} & \frac{\partial^{2} L_{c}}{\partial y \partial v} \\
\frac{\partial^{2} L_{c}}{\partial u \partial x} & \frac{\partial^{2} L_{c}}{\partial u \partial y} & \frac{\partial^{2} L_{c}}{\partial u^{2}} & \frac{\partial^{2} L_{c}}{\partial u \partial v} \\
\frac{\partial^{2} L_{c}}{\partial v \partial x} & \frac{\partial^{2} L_{c}}{\partial v \partial y} & \frac{\partial^{2} L_{c}}{\partial v \partial u} & \frac{\partial^{2} L_{c}}{\partial v^{2}}
\end{array}\right),
$$

and $\mathbf{D}$ is a diffusion tensor to be defined according to the regularization goals. This formulation extends the $2 \mathrm{D}$ anisotropic diffusion [4] to 4D light fields.

Each eigenvector $\nu_{i}$ in (4) is responsible for setting a direction for the diffusion process. Since these vectors are eigenvectors of the 4D light field structure tensor, these orientations depend on the local structure properties. In order to best preserve the structures in the $4 \mathrm{D}$ ray space, when running the diffusion, it is very important to understand how these orientations capture anisotropy in the $4 \mathrm{D}$ ray space, which is much less straightforward than in 2D.

\section{Influence of eigenvectors on the diffusion tensor}

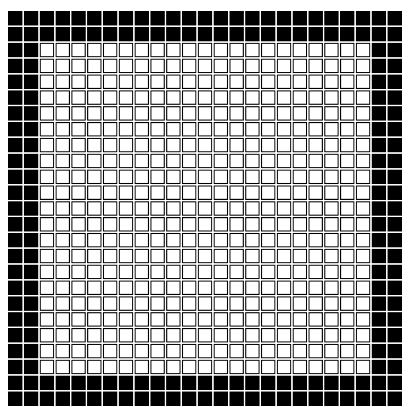

(a)

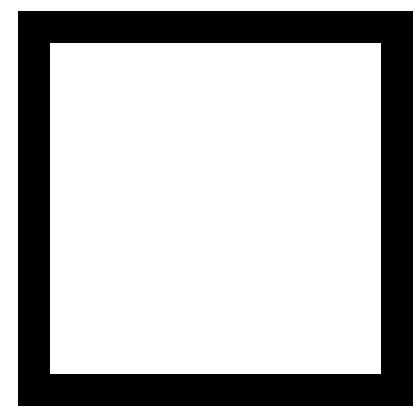

(b)
Fig. 1: Tesseract as a 4D light field toy example: (a) Set of $25 \times 25$ light field views; (b) Subaperture image of $25 \times 25$ pixels image containing a square at the center of $21 \times 21$ pixels for the central views (fully black image at the periphery of the light field).

To understand how each eigenvector influences the diffusion process, we first consider a 4D cube, i.e., a tesseract, as illustrated in Fig. 1, where we show its 2D projection. Because of its symmetry and of the fact that such shape contains faces, edges, and corners, it provides a good example to illustrate how the eigenvector oriented diffusion behaves on 4D structures. Fig. 2 shows the effect of the diffusion process when the diffusion tensor is based on a single eigenvector. In 
Fig. 2a, the diffusion tensor is based on the fourth eigenvector $\nu_{4}$, hence follows a direction corresponding to the highest degree of singularity in the 4D space. For the tesseract, these degrees of singularity are located at the hyper-corners, i.e., at the corners of corners in the 2D projection. The diffusion process therefore smoothes these hyper-corners, i.e., rounds the squares at angular corners of the tesseract. In Fig. 2d, the diffusion tensor is based on the third eigenvector $\nu_{3}$ that defines a direction corresponding either to the corners of the edges, or to the edges of the corners. In Fig. $2 \mathrm{~g}$, the second eigenvector $\nu_{2}$ has a direction corresponding to any 2D corner in the 4D space or to edges of edges. We can see that the diffusion along $\nu_{2}$ leads to a more global smoothing of the original structure as compared to the diffusion along $\nu_{3}$. The same applies for $\nu_{1}$ regarding $\nu_{2}$. One can note that diffusing along $\nu_{2}, \nu_{3}$ and $\nu_{4}$ using $\mathbf{D}=\nu_{2} \nu_{2}^{T}+\nu_{3} \nu_{3}^{T}+\nu_{4} \nu_{4}^{T}$ tends to create a 4D hypersphere, i.e., a glome.

\section{Setting the diffusion tensor parameters}

The diffusivity coefficients $\beta_{i}$ introduced in (4) control the strength of the diffusion in the eigenvectors orientations, hence the amount of anisotropy. Therefore, their definition is mostly responsible of the diffusion steering, regarding both its intensity and its direction. Indeed, the inequality of the $\beta_{i}$ coefficients produces the anisotropy that is needed to strengthen the structures in most of the inverse problems applications we consider here. In some cases, as in the following section, it is relevant to express these coefficients as functions $f_{i}$ depending on the quantity of structure $\psi$. This means the diffusion steering, in addition of being expressed on a basis defined by structures (the eigenvectors), is also driven by the structures intensity. We define this intensity such as: $\psi=\sqrt{\sum_{i=1}^{4} \lambda_{i}}$, which is considered to be a good measure of local variation [32].

In the end, depending on the application, the diffusion can be either driven by constants: $\beta_{i}=A_{i}$ as for spatial and angular interpolation as we will see later, or by the local structures: $\beta_{i}=f_{i}(\psi)$, as we will detail next.

\section{DENOISING}

In order to preserve the coherence of the light field structures, while removing incoherent information such as noise, the function $f_{i}$ must verify certain properties. First, the diffusion should be isotropic in regions where there is no strong apparent direction, i.e.:

$$
\lim _{\psi \rightarrow 0} \mathbf{D}=a \mathbf{I} \Rightarrow f_{1}(0)=f_{2}(0)=f_{3}(0)=f_{4}(0) .
$$

Conversely, in regions where structures are clearly appearing, no diffusion should be performed in order to preserve geometry, i.e.:

$$
\lim _{\psi \rightarrow \text { inf }} \mathbf{D}=0 \Rightarrow f_{1}(\psi)=f_{2}(\psi)=f_{3}(\psi)=f_{4}(\psi)=0 .
$$

Also, the diffusion must be performed along directions describing the local structures. The singularity of structures is described by the eigenvectors having the lowest eigenvalues. The more structure there is, the more the lowest eigenvalued

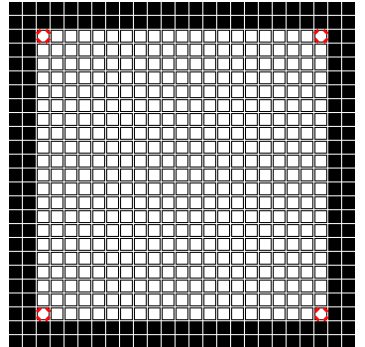

(a)

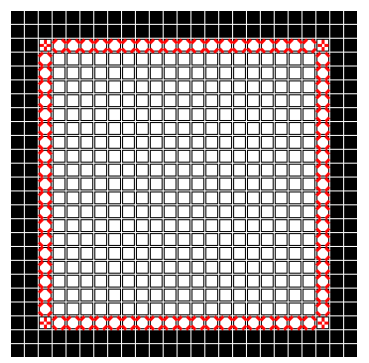

(d)

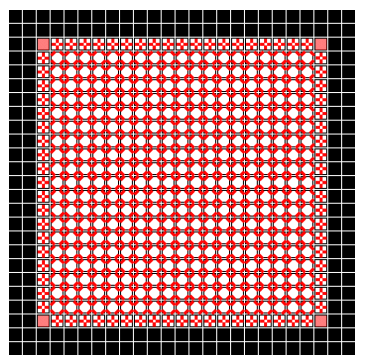

(g)

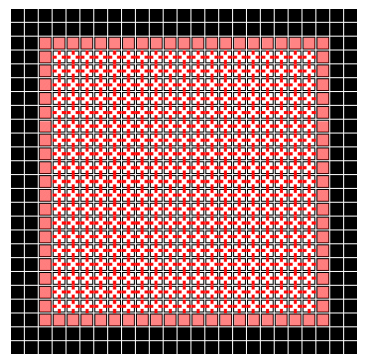

(j)

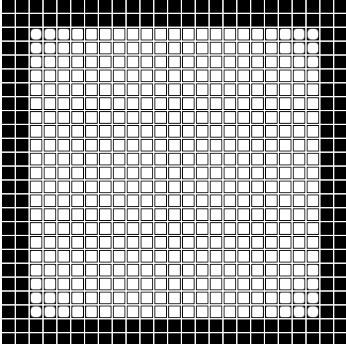

(b)

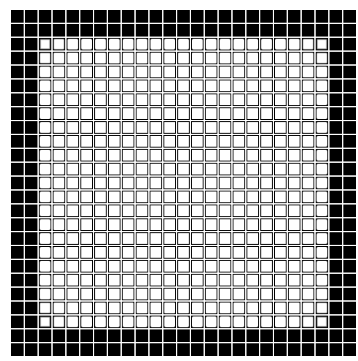

(e)

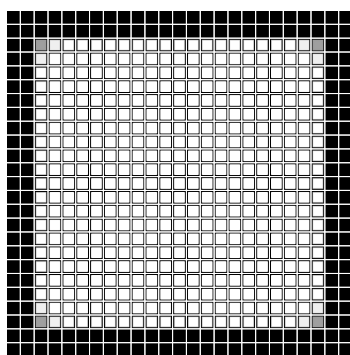

(h)

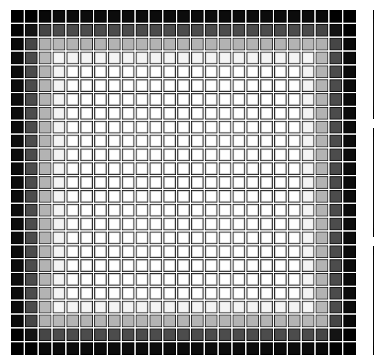

(k)

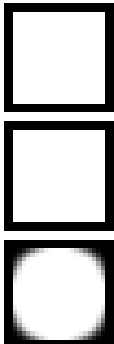

(c)

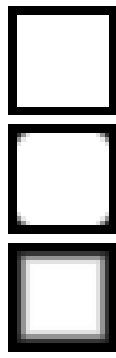

(f)

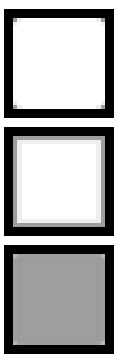

(i)

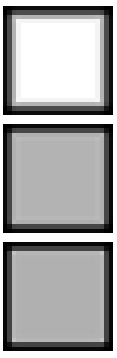

(1)
Fig. 2: Tesseract diffusion. (a-c) Tensor is constructed using $\nu_{4}$. (d-f) Tensor is constructed using $\nu_{3}$. (g-i) Tensor is constructed using $\nu_{2}$. (j-1) Tensor is constructed using $\nu_{2}$. $(\mathrm{a}, \mathrm{d}, \mathrm{g}, \mathrm{j})$ Initialization and diffusion orientation in red. (b,e,h,k) Diffusion at early iterations. (c,f,i,l) Close-up on (from top to bottom) center view $[\mathrm{u}=12, \mathrm{v}=12]$, edge view $[\mathrm{u}=22, \mathrm{v}=12]$ and corner view $[\mathrm{u}=22, \mathrm{v}=22]$.

eigenvectors must drive the diffusion. When the structure is clearly shaped, the diffusion should only correct structure singularities with the highest degree. Applying such reasoning to the eigenvectors, we obtain the condition:

$$
f_{1}(\psi) \leq f_{2}(\psi) \leq f_{3}(\psi) \leq f_{4}(\psi) .
$$

We therefore propose the function $f_{i}$ meeting the conditions of equations (7), (8) and, (9):

$$
f_{i}(\psi)=e^{-\left(\frac{\psi}{\rho_{i}}\right)^{\gamma}}, \text { with } \rho_{1} \leq \rho_{2} \leq \rho_{3} \leq \rho_{4}
$$

where $\rho_{i}$ is a parameter related to a quantity of local variation $\psi$ at which diffusion along $\nu_{i}$ will importantly change 
the intensity, and $\gamma$ is a parameter defining the exponential behaviour of the function. This parametrization allows tuning the diffusion process on the basis of structure properties. The parameters $\gamma$ and $\rho_{i}$ need however to be adjusted according to the application. Below, we illustrate the effect of these parameters in the context of light field denoising.

\section{A. Model tuning}

The best set of diffusion model parameters $\rho_{i}$ may depend on the light field and on the desired smoothing intensity. Finding the best values for this set of parameters $\rho_{i}$ may therefore be a tedious task. Below, we propose a simplified parametrization to facilitate the model tuning.

Instead of parameterizing independently each parameter $\rho_{i}$, we propose the following expression:

$$
\rho_{i}=\rho_{r} \alpha^{i-4},
$$

which allows a simplification of the model, being left with only two parameters. The first one, $\rho_{r}$, is related to a local variation intensity. The second one, $\alpha$, is related to the amount of anisotropy for the diffusion. Fig. 3 plots different functions $f_{i}(\psi)$ parameterized by $\rho_{i}$, taking $\rho_{r}=0.4$ and $\alpha=2$. Below, we study the influence of these parameters on the denoising process. For each experiment, we set $\gamma=2$, and the same time step of 0.1 to better show the influence of the parameter $\rho_{r}$ on the denoising process.

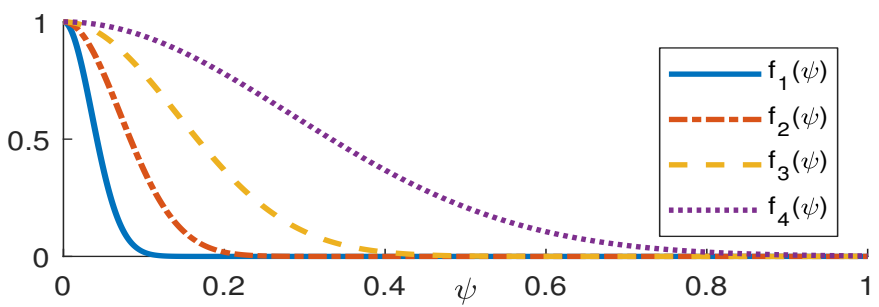

Fig. 3: Functions $f_{i}(\psi)$ controlling the smoothing strength of the diffusion tensor along orientations defined by the four eigenvectors $\nu_{i}$, with $\rho_{r}=0.4$ and $\alpha=2$.

1) Model range and noise intensity: Fig. 4 shows the evolution of the PSNR along the iterations of the model integration, when varying the parameter $\rho_{r}$, with different levels of noise. Note that the PSNR is the Peak Signal to Noise Ratio computed as $10 \log _{10} \frac{1}{M S E}$, where the MSE is the mean square error between all the original (with no noise) and all the denoised views over the three RGB color components. The light field used in this experiment is Bike of the EPFL dataset [33]. The integration time $t$ is related to the number of iterations $n$ by the time step $d t=0.1$ such as: $t=n d t$. The integration carried out to solve (5) is performed using the Runge-Kutta 4 scheme. Fig. 4a shows that when no noise is applied to the light field, higher values of $\rho_{r}$ yield higher deterioration (smoothing) of the light field. Fig. $4 \mathrm{~b}$ and $4 \mathrm{c}$, show that, for low noise $\left(\sigma=\frac{10}{255}, \sigma=\frac{40}{255}\right)$, a low value of $\rho_{r}$ (respectively 0.08 and 0.24 ) prevents the model to damage the light field structures. On the other hand, a low value of $\rho_{r}$ is not suitable for high noise levels as shown in Fig. 4d, where the model is unable to diffuse along the structures, hence many

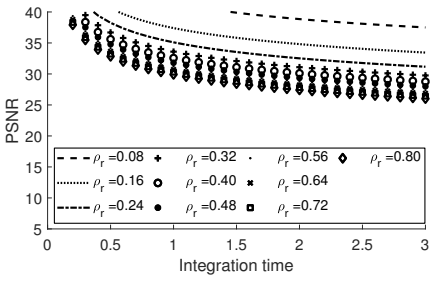

(a)

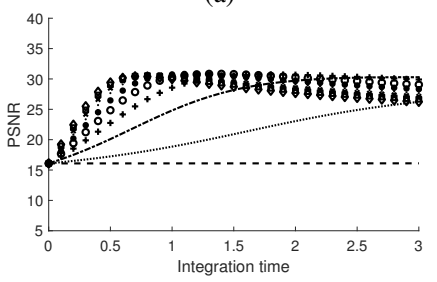

(c)

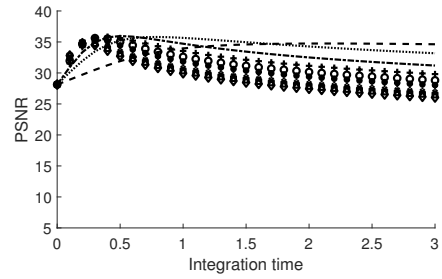

(b)

(d)

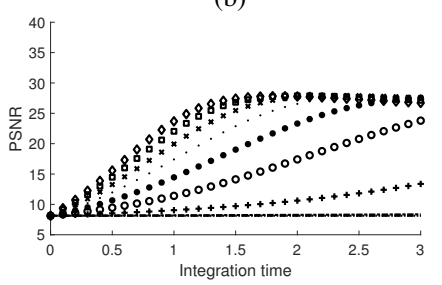

Fig. 4: Denoising behaviour for different noise levels applied to the Bikes light field. Each curve represents the PSNR evolution along the diffusion iterations, for different values of the parameter $\rho_{r}$, in the cases of (a) No noise; (b) additive Gaussian noise with $\sigma=\frac{10}{255}$; (c) $\sigma=\frac{40}{255}$; and (d) $\sigma=\frac{100}{255}$. The parameter $\alpha$ controlling the anisotropy is set to 2 .

iterations are needed to remove the noise. For high values of $\rho_{r}$, we can observe a more efficient behaviour regarding noise reduction. But in this case, the model can damage the structures (more than with low values of $\rho_{r}$ ), as we can see when the number of iterations increases.

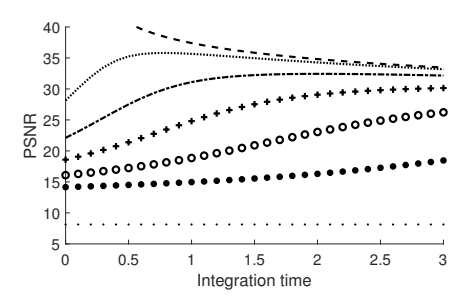

(a)

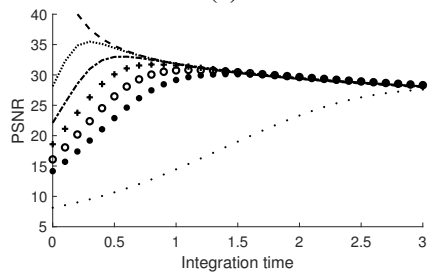

(c)

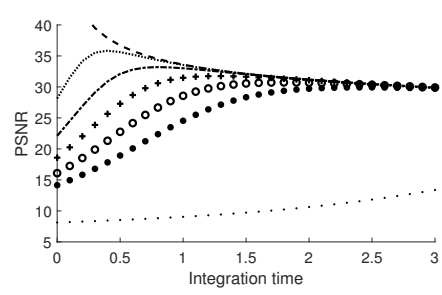

(b)

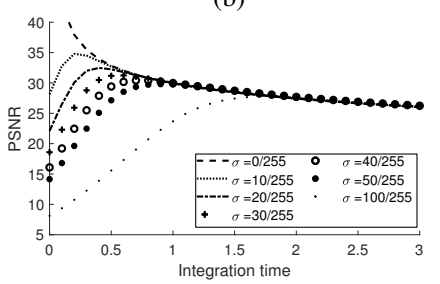

(d)
Fig. 5: Denoising behaviour for different models applied to the Bike light field of the EPFL dataset [33]. Each curve represents the PSNR evolution along the diffusion iterations for different noise standard deviations $\sigma_{G}$, using a model parameterized by (a) $\rho_{r}=0.16$; (b) $\rho_{r}=0.32$; (c) $\rho_{r}=0.48$; (d) and $\rho_{r}=0.80$. The parameter $\alpha$ controlling the anisotropy is set to 2 .

Fig. 5 shows, in a symmetric manner compared with Fig. 4, the evolution of the PSNR along the iterations, when varying the levels of noise, with different values of the parameter $\rho_{r}$. Fig. 5b-d show that, for different values of the parameter $\rho_{r}$, when increasing the number of iterations, the model converges to the same PSNR curve, at both low and high noise levels. This shows that, even for very different initial noise intensities, a same denoising model (with same parameters) will tend to reshape the light field in almost the same fashion, provided 
$\rho_{r}$ or the number of iterations is high enough. In other words, the noise is cleaned more or less quickly while the model is on its way to its inherent convergence state. In the following denoising experiments, we chose to set the parameter $\rho_{r}$ to 0.4 , which appeared to be a good trade-off between preserving data structures and efficiency in terms of noise removal.

2) Anisotropy: We now study how the coefficient $\alpha$ controlling the amount of anisotropy influences the denoising process. Fig. 6d-f shows denoising results for $\alpha$ set to 1 (isotropic), 2, and 5, after 20 iterations, using the StonePillarsOutside light field. Even if this number of iterations may not yield the best reachable PSNR for each of the $\alpha$ values, it allows analyzing the model behaviour when varying the parameter $\alpha$. While the isotropic denoising yields too blurry images (see Fig. 6d), a high value of $\alpha$ tends to sharpen too much the details, even to structure the noise instead of removing it (see Fig. 6f).

As the anisotropy increases (Fig. 6i), the best PSNR is obtained for a higher number of iterations, as we can see in Fig. 6c, highlighted by the red line. Increasing the time step can lower the number of iterations, thus the computation time, but can affect the stability of the model integration. Indeed, the non-linearity of the model is likely to increase with its anisotropy. In the end, a trade-off has to be found depending on how fine one wants to recover texture details, and how fast the denoising reaches the best result. We have observed experimentally that setting $\rho_{r}$ to 0.4 and $\alpha$ to 2 was yielding a good compromise between efficiency, sharpness and number of iterations.

\section{B. Denoising results with the tesseract toy example}

In order to illustrate the behaviour of the denoising model, we propose to apply it on the tesseract toy example presented above. The goal is to observe how the proposed denoising model behaves on a light field containing both very high and very low variations in the 4D space. Fig. 7 shows denoising results, after 20 iterations, with both the $2 \mathrm{D}$ regularization method described in [8] applied independently on each view, and the proposed 4D anisotropic diffusion. One can observe that the proposed diffusion performs well in terms of denoising compared with the 2D approach of [8]. The 4D angularities of the tesseract are being smoothed in every dimension.

\section{Denoising results with real light fields}

In order to assess the denoising performance of the 4D anisotropic diffusion with the proposed model on real light fields, in comparison with state of the art methods, e.g., with [2], we considered the same 12 light fields of the EPFL dataset [33]. The light fields have been extracted from the raw captures using Dansereau's Matlab light field toolbox [34] in the same conditions as in [2].

Because of the strong irregularities in the angular dimension resulting from the vignetting effect, we chose to denoise only the subapertures that are not affected by the later and therefore limit the size of light fields to $11 \times 11$ central views. Indeed, because of its regularization properties, we will show later that our method also corrects artefacts at angular corners (i.e., the vignetting effect), hence the measured PSNR, when

\begin{tabular}{|c||c|c|c|c|c||}
\hline Method & $\sigma=\frac{10}{255}$ & $\sigma=\frac{20}{255}$ & $\sigma=\frac{30}{255}$ & $\sigma=\frac{40}{255}$ & $\sigma=\frac{50}{255}$ \\
\hline HF4D & 31.07 & 25.80 & 22.61 & 20.34 & 18.59 \\
\hline BM3D & 35.42 & 32.85 & 31.36 & 30.25 & 29.32 \\
\hline BM3D Epi & 36.09 & 33.48 & 31.91 & 30.71 & 29.67 \\
\hline VBM4D & 36.08 & 33.52 & 31.92 & 30.67 & 29.63 \\
\hline VBM4D Epi & 36.13 & 33.51 & 31.93 & 30.72 & 29.72 \\
\hline LFMB5D 1st step & 34.39 & 32.81 & 31.68 & 30.74 & 29.91 \\
\hline LFMB5D 2nd step & $\mathbf{3 6 . 5 0}$ & $\mathbf{3 4 . 2 1}$ & $\mathbf{3 2 . 8 7}$ & $\mathbf{3 1 . 8 4}$ & 30.99 \\
\hline$\rho_{r}=0.4, \alpha=2$ & 36.13 & 33.88 & 32.67 & $\mathbf{3 1 . 8 4}$ & $\mathbf{3 1 . 1 9}$ \\
\hline
\end{tabular}

TABLE I: Comparison of light field denoising methods (courtesy of [2] for the PSNR values in $\mathrm{dB}$ of the reference methods). A visual comparison can be found in Fig. 10.

considering all the views, would not only reflect the denoising performance.

In the experiments, we chose to use the same model parameterized by $\rho_{r}=0.4, \alpha=\gamma=2$, and the tensor smoothing is set to $\omega=1.1$ in the spatial and angular directions. This parameterization gives a denoising model of relatively low anisotropy, but allows the removal of noise in a reasonable number of iterations. One iteration takes 7 minutes to compute on a i7-6600U without any parallelization, and best PSNR values are usually obtained from 4 iterations to 20 depending on the light field and the model. Note that the computation time for each iteration is not scene dependent and is the same for light fields of same dimension. For comparison, LFBM5D performs approximately in 7 hours on an octo core processor (but on $15 \times 15$ light fields). On a 10 iterations basis, our method gives an estimated 26 times faster result. Detailed results can be found online ${ }^{1}$ to show angular consistency. In Fig. 8, we observe that our model is able to remove low noise while keeping fine original structures. Our model is also able to deal with high levels of noise as shown in Fig. 9 and 10. In Fig. 10, we can see that the results qualitatively match the best existing denoising method. In Table I we can see that the denoising model we used outperforms other methods for a high level of noise, while comparing well for low levels of noise.

Fig. 11 shows an example of denoising result with a light field captured under low illumination conditions. In this experiment, the denoising model is parameterized as a small variant of the expression proposed in (11), i.e. by setting the model parameters as $\rho_{i}=\rho_{i+1} \alpha^{i-4}$. Our method is able to remove noise due to poor capture conditions. We also provide in Tab. II additional restoration results to show the efficiency of the proposed approach in other conditions than additive Gaussian noise. The restoration of light field quantized using the Floyd-Steinberg dithering method yields particularly good results, as presented in Fig. 12. Indeed, the dithering preserves image structures and color gradients, thus allowing more efficient reconstruction using the tensor diffusion approach.

\section{Influence of the number of views}

It is interesting to study to which extent the light field structure is retrieved when the number of light field views increases. In the following experiments, we use the original parameterization model proposed in (11), with $\rho_{r}=0.4$ and $\alpha=2$. Fig. 13 shows how the error evolves along the iterations

\footnotetext{
${ }^{1}$ http://clim.inria.fr/research/AD4D
} 


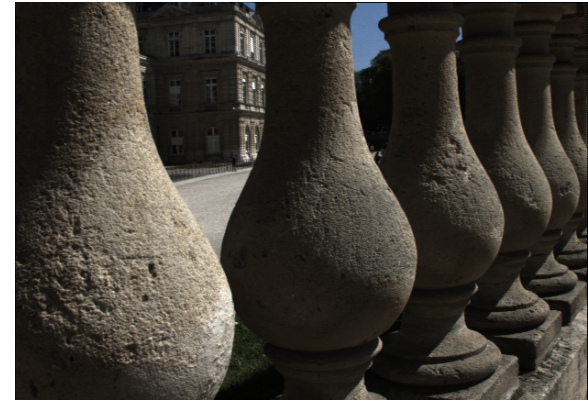

(a) Original (center view)

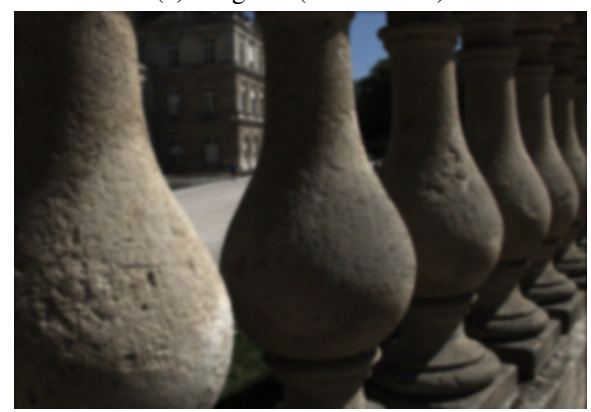

(d) $\alpha=1,20$ iterations, PSNR $26.21 \mathrm{~dB}$.

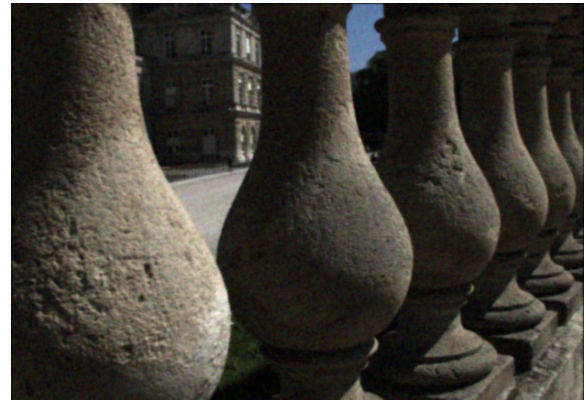

(g) $\alpha=1,6$ iterations (best), PSNR $28.37 \mathrm{~dB}$.

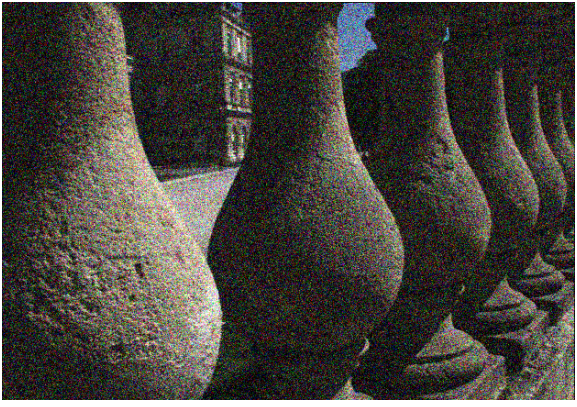

(b) Noised, $\sigma=\frac{50}{255}$, overall PSNR $14.15 \mathrm{~dB}$.

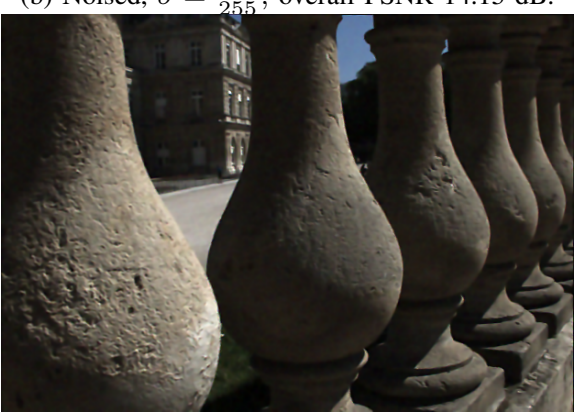

(e) $\alpha=2,20$ iterations, PSNR $28.69 \mathrm{~dB}$

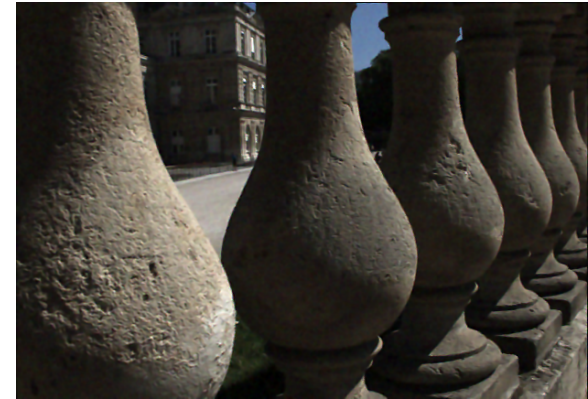

(h) $\alpha=2,16$ iterations (best), PSNR $30.31 \mathrm{~dB}$.

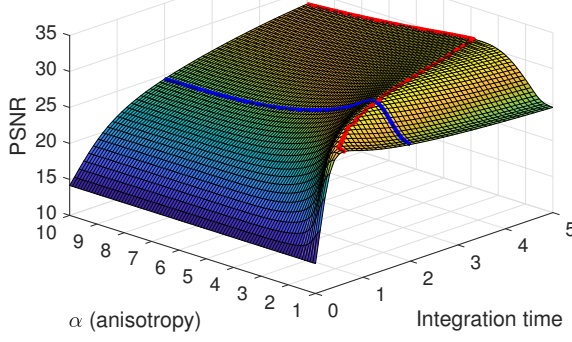

(c) Overall PSNR evolution as function of $\alpha$.

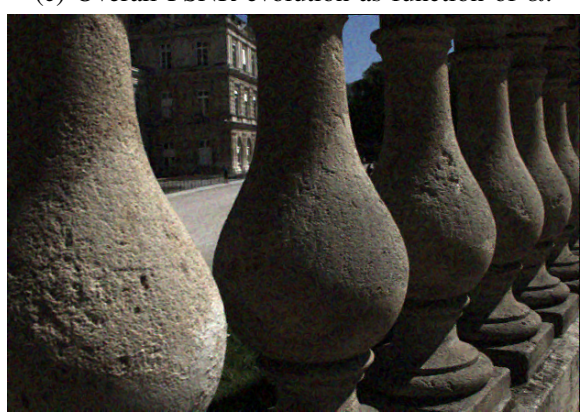

(f) $\alpha=5,20$ iterations, PSNR $26.18 \mathrm{~dB}$

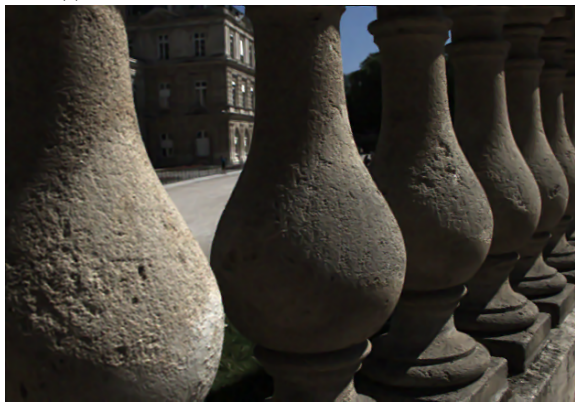

(i) $\alpha=5,50$ iterations (best), PSNR $30.58 \mathrm{~dB}$.

Fig. 6: Influence of the anisotropic coefficient $\alpha$ on light field denoising using StonePillarsOutside of the EPFL dataset [33]. (a) Original center view; (b) center view with additive white Gaussian noise $\left(\sigma=\frac{50}{255}\right)$; (c) Influence of the anisotropic coefficient $\alpha$ on denoising efficiency. (d-f) Denoising results with parameter values on the blue line in Fig. (c): (d) Isotropic diffusion after 20 iterations. Structures are smoothed, no matter what their directions are. (e) Important structures are preserved, while textures are flattened. (f) Structures are preserved, thus yielding finer textures. However, the noise is getting structured in homogeneous areas (ground, sky, shades) instead of being isotropically smoothed, thus increasing error. (g-i) Best PSNR (red line in (c)).

\begin{tabular}{|c|c|c|c|c|c|c|c|c|c|c|c|c|}
\hline & \multicolumn{3}{|c|}{$\begin{array}{c}\text { Poisson noise } \\
\text { standard deviation ratio }\end{array}$} & \multicolumn{3}{|c|}{$\begin{array}{c}\text { Uniform quantization } \\
\text { color levels per channel }\end{array}$} & \multicolumn{4}{|c|}{$\begin{array}{l}\text { Dithering quantization } \\
\text { color levels per channel }\end{array}$} & \multicolumn{2}{|c|}{$\begin{array}{l}\text { K-means quantization } \\
\text { number of colors }\end{array}$} \\
\hline & 0.1 & $\mid 0.01$ & 0.001 & 8 & 16 & 32 & 2 & 4 & 8 & 16 & 32 & 64 \\
\hline & 15.87 & 25.87 & 35.86 & 28.05 & 34.60 & 40.93 & 9.11 & 18.22 & 25.73 & 32.8 & 32.0 & 34.19 \\
\hline Restor & 30.82 & 34.75 & 39.55 & 30.67 & 37.22 & 42.33 & 28.83 & 33.06 & 36.02 & 38.8. & 33.39 & 35.39 \\
\hline Iterations & 20 & 5 & 1 & 5 & 2 & 1 & 39 & 9 & 4 & 2 & 2 & 2 \\
\hline
\end{tabular}

TABLE II: Light field restoration with different types of noise. The PSNR values (in dB) are averaged over the 12 EPFL light fields [33]. We also give the rounded number of iterations at which the maximum PSNR is reached. The denoising model is parameterized by $\rho_{r}=0.4$ and $\alpha=2$.

for different numbers of views. In Fig. 13a, we can see that the maximum overall PSNR increases with the number of views (up to $9 \mathrm{x} 9$ views). Starting from $11 \times 11$ views, the error increases because of the vignetting effect of the light-field capturing device. Indeed, as mentioned in section IV-C, light fields captured with Lytro Illum cameras are impacted by lens deformations producing angular inconsistencies. For a high number of views (higher than $11 \times 11$ in the case of Lytro Illum captures), these irregularities are being corrected by the denoising process, and tend to increase the difference with the original light field.

For $13 \times 13$ views, the vignetting effect starts to clearly appear (also see Fig. 14e,f and Fig. 15). The strong intensity variations between vignetted (almost entirely black) and inner views produce angular structures which are difficult to properly smooth. Increasing the anisotropic coefficient $\alpha$ could enhance these contours, but at the expense of sharpening the noise in other regions. Such problem can also be viewed in Fig. 7f (middle and bottom), where the denoising process yields smoothing of structures at angular edges and corners. 


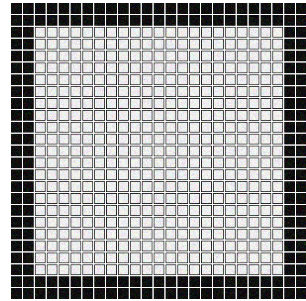

(a) PSNR $16.09 \mathrm{~dB}$

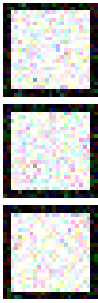

(b)

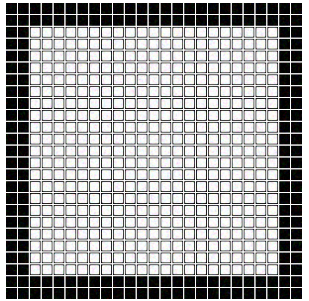

(c) PSNR $22.56 \mathrm{~dB}$

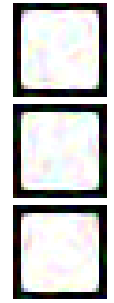

(d)

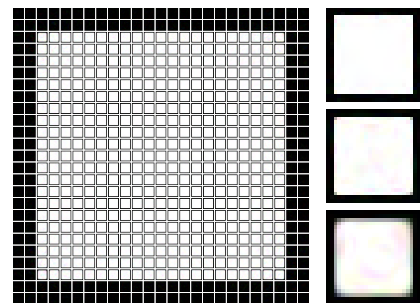

(e) PSNR $30.92 \mathrm{~dB}$

(f)

Fig. 7: Denoising of the Tesseract toy example. (a,b) Gaussian noise added to tesseract example (standard deviation $\sigma=\frac{40}{255}$ ). (c,d) After 20 iterations, using the $2 \mathrm{D}$ denoising method proposed in [8]. (e,f) After 30 iterations using the proposed 4D denoising model. PSNR computed over all images.

This side effect is further discussed in this section.

If we now consider only the central views of the light field, we can see in Fig. 13b that the maximum PSNR value is almost the same from $5 \times 5$ to $15 \times 15$ views. However, as the number of views increases, this maximum is reached with fewer iterations because of the larger distance to angular edges, and consequently the lower angular boundary effect. On the other hand, when the number of views increases, more angular inconsistencies are corrected, and eventually propagated to the center view, causing the PSNR to decrease.

To better see that effect, Fig. 14 shows the PSNR obtained after denoising for each view of the light field. We can see that, at the corner views, the PSNR decreases when the number of views increases. One can also observe in Fig. 14d that the values of PSNR are not symmetric around the angular axes, values at the angular coordinates $[0,0]$ being lower than others. This can be explained by the vignetting effect and inconsistencies between micro-lenses.

As mentioned above, for $13 \times 13$ views (see Fig. 14e), the vignetting effect is clearly visible. The error on the corner views of the light field becomes more important than in inner views. Indeed, as we can observe in Fig. 15, the denoising process yields structure smoothness likely to print scene structures on vignetted views, thus increasing the error at this angular location. This effect can be lowered by increasing the anisotropic coefficient $\alpha$, so the diffusion of the denoising model can better fit the contrasted structures, such as the ones the vignetting produces in the angular space. Eventually, for $15 \times 15$ views (see Fig. 14e), we observe a high PSNR rebound for the angular corner views which are almost fully black with no neighboring contrasted structures.

\section{E. Sparsity}

The proposed method can be challenged when the angular consistency between views decreases. Indeed, sparse light

fields, with large baselines, do not always provide continuity of structures in the angular dimension. Fig. 16a shows a denoising example on a light field from the Stanford dataset [36]. The foreground of the scene (highlighted by the bottom white line) presents sharp structures in the angular space because of the high disparity at this location. Indeed, in the corresponding epipolar plane image, we can see that the structures are strongly aliased, with little inter-view continuity.

During the denoising process (see Fig. 16c), the sharp structures are difficult to preserve. Any smoothing of structures in the angular space, also leads to significant blurriness of the views. This effect is related to the toy experiment presented in Fig. 7, and more specifically to the f-bottom view. Indeed, spatial structures with high inter-view disparity corresponds to hyper corners of the tesseract. These 4D singularities are difficult to denoise while keeping intact their complex structure.

\section{F. Non-Lambertian surfaces}

Light reflections during light field captures can produce complex 4D geometries. We propose to apply our denoising method on a light field containing non-Lambertian surfaces. In Fig. 18, we can see that the reflected structures on the water are correctly denoised.

\section{Miscellaneous applications}

The proposed 4D tensor-based anisotropic diffusion can be a useful tool for various applications, beyond denoising. Here, we consider view (or angular) interpolation, as well as spatial interpolation. The goal is to highlight the fact that our approach deals with light fields as 4D volumes, and can perform well in different application contexts.

\section{A. View interpolation}

We use instead the proposed anisotropic diffusion approach to estimate intermediate views by strengthening structures across views. While for the denoising application, isotropic diffusion is required to remove noise in homogeneous areas, the diffusion process for view interpolation is required to preserve existing structures of original views, while creating new intermediate views. By setting the values of the parameters $\beta_{i}$ of (4) as $\beta_{1}=0, \beta_{2}=0.25, \beta_{3}=0.5$, and $\beta_{4}=1$, we obtain a regularization model suitable for the angular interpolation problem. The increasing values of beta are explained by the goal of first propagating the strongest structures (hence with a stronger diffusivity coefficient) and then the homogeneous regions. The orientations of the strongest structures are given by the $4^{\text {th }}$ eigenvector and correspond in the view interpolation problem to strong luminance variations in both angular dimensions due to the missing views to be interpolated. Before computing the tensor, and only for the purpose of the tensor computation, we apply a convolution with a Gaussian kernel on the light field as $L_{w}=L * G_{w}$, with $w=\left[w_{x}, w_{y}, w_{u}, w_{v}\right]$. In the following experiments, we set $w_{x}=w_{y}=0$ and $w_{u}=w_{v}=0.8$. For the view interpolation problem, the structure tensor is used to find coherent orientations in the 


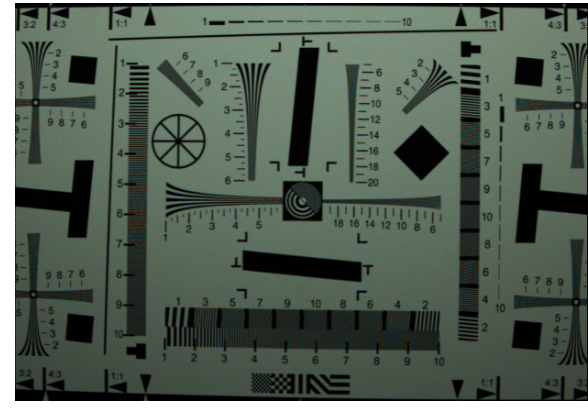

(a) Original.

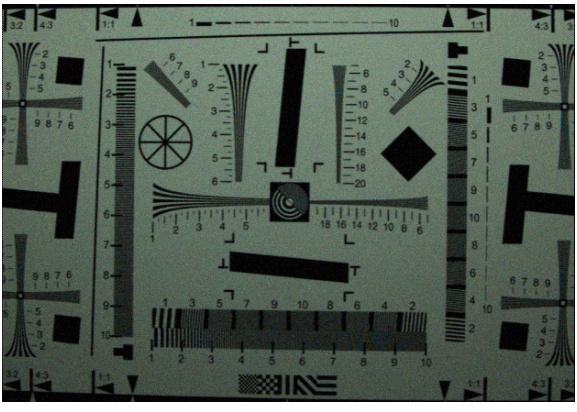

(b) Overall PSNR $28.13 \mathrm{~dB}$.

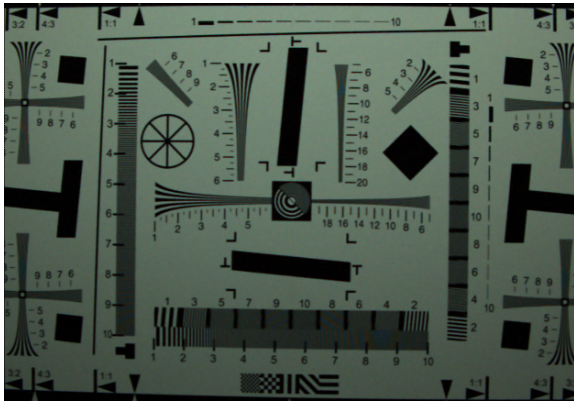

(c) Overall PSNR $35.10 \mathrm{~dB}$.

Fig. 8: Denoising of Iso Chart 12 light field. Center views. (a) Original. (b) Gaussian noise of $\sigma=\frac{10}{255}$. (c) Using our method, 4 iterations.

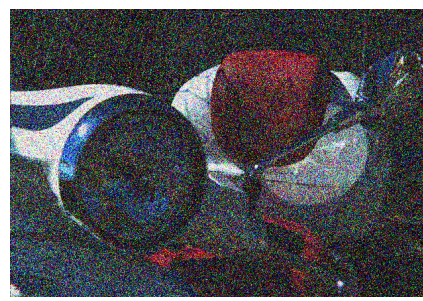

(a)

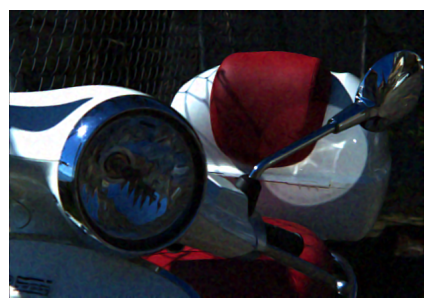

(b)

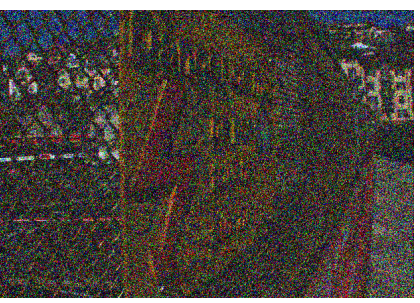

(c)

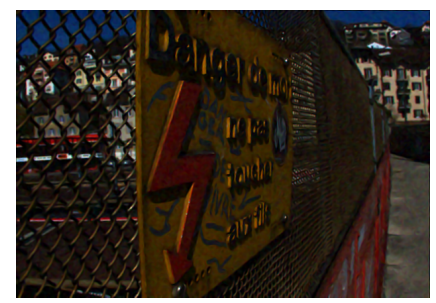

(d)

Fig. 9: 4D tensor driven diffusion is able to denoise highly damaged light fields. Center views for Vespa and DangerDeMort from the EPFL dataset [33]. (a,c) With Additive Gaussian noise $\left(\sigma=\frac{100}{255}\right.$, overall PSNR $8.13 \mathrm{~dB}$ ); (b,d) After denoising giving a PSNR of $29.31 \mathrm{~dB}$ (b) and $28.50 \mathrm{~dB}(\mathrm{~d})$.

\begin{tabular}{|c|c|c|c|c|c|c|c|c|c|c|c|c|}
\hline \multirow{3}{*}{ Light field } & \multicolumn{6}{|c|}{ Integer magnification, factor 2} & \multicolumn{6}{|c|}{ Integer magnification, factor 3} \\
\hline & \multirow{2}{*}{\begin{tabular}{|l|} 
Nearest \\
\end{tabular}} & \multicolumn{2}{|c|}{ RGB } & \multicolumn{3}{|c|}{ Luminance } & \multicolumn{3}{|c|}{ RGB } & \multicolumn{3}{|c|}{ Luminance } \\
\hline & & Bicubic & Diffusion & Nearest & Bicubic & Diffusion & Nearest & Bicubic & Diffusion & Nearest & Bicubic & Diffusion \\
\hline Bikes & 24.54 & 26.98 & 28.43 & 25.32 & 28.25 & 29.58 & 23.08 & 24.03 & 26.05 & 23.81 & 24.79 & 26.80 \\
\hline Bench in Paris & 20.71 & 22.97 & 23.70 & 21.10 & 23.55 & 24.16 & 19.45 & 20.08 & 21.22 & 19.83 & 20.48 & 21.56 \\
\hline Friends 1 & 25.98 & 27.52 & 29.17 & 26.79 & 28.26 & 30.03 & 24.44 & 25.14 & 27.75 & 25.16 & 25.83 & 28.63 \\
\hline Sphynx & 24.83 & 26.65 & 28.54 & 25.40 & 27.29 & 29.30 & 23.53 & 23.92 & 26.31 & 24.05 & 24.43 & 26.95 \\
\hline Bee 2 & 25.62 & 28.20 & 29.17 & 28.02 & 31.65 & 32.52 & 24.50 & 25.51 & 27.14 & 26.80 & 28.06 & 29.83 \\
\hline Duck & 21.67 & 24.66 & 24.91 & 22.78 & 25.81 & 26.19 & 20.49 & 21.09 & 22.43 & 21.56 & 22.21 & 23.55 \\
\hline Fruits & 24.55 & 27.61 & 28.61 & 25.40 & 28.83 & 29.79 & 23.49 & 24.17 & 26.12 & 24.31 & 25.03 & 27.05 \\
\hline Rose & 27.96 & 30.73 & 32.20 & 29.05 & 31.87 & 33.45 & 26.56 & 27.45 & 29.80 & 27.54 & 28.29 & 30.84 \\
\hline Mini & 24.30 & 26.27 & 27.64 & 24.98 & 26.97 & 28.34 & 23.08 & 23.46 & 25.52 & 23.73 & 24.10 & 26.17 \\
\hline Buddha & 29.90 & 33.19 & 33.55 & 30.07 & 33.37 & 33.72 & 28.51 & 29.47 & 30.88 & 28.66 & 29.63 & 31.03 \\
\hline Cone Head & 33.11 & 36.64 & 35.54 & 35.57 & 39.12 & 38.51 & 32.03 & 32.59 & 33.63 & 34.51 & 35.16 & 36.29 \\
\hline Monas & 29.28 & 32.94 & 32.92 & 30.05 & 33.49 & 34.03 & 28.15 & 28.90 & 30.54 & 29.91 & 29.58 & 31.44 \\
\hline Watch & 30.16 & 37.35 & 36.18 & 30.16 & 37.35 & 36.18 & 28.96 & 30.77 & 32.11 & 28.96 & 30.77 & 32.11 \\
\hline Papillon & 32.00 & 35.62 & 37.50 & 32.01 & 35.75 & 37.82 & 30.86 & 32.20 & 34.65 & 30.87 & 32.22 & 34.84 \\
\hline Still Life & 22.61 & 24.79 & 25.41 & 22.72 & 24.87 & 25.50 & 21.01 & 21.20 & 22.75 & 21.10 & 21.27 & 22.82 \\
\hline Average & 26.48 & 29.47 & 30.23 & 27.29 & 30.43 & 31.27 & 25.21 & 26.00 & 27.79 & 26.05 & 26.79 & 28.66 \\
\hline $\begin{array}{l}\text { Average increment } \\
\text { from bicubic }\end{array}$ & -2.99 & 0 & +0.76 & -3.14 & 0 & +0.84 & -0.79 & 0 & +1.79 & -0.74 & 0 & +1.87 \\
\hline
\end{tabular}

TABLE III: PSNR using integer magnification setup, and for factor 2 and 3. High increment from nearest neighbor to bicubic for magnification factor 2 is due to the misalignment of the nearest neighbor method for even factors.

angular $u$ and $v$ dimensions, hence is smoothed only in these dimensions.

Fig. 19 shows the results obtained with two light fields that have been sub-sampled with a factor of 2 . The figures show the reconstructed views along the iterations, thus illustrating the diffusion effect. Most contrasted structures appear first, and homogeneous areas are progressively filled. A lower PSNR is obtained for the Desktop light field due to angular inconsistencies. The noise of the subaperture images is also corrected, yielding a lower PSNR, when computed with respect to the noisy input images. The computation can be speeded up by increasing $\beta_{2}$ to tend towards orthogonal isotropy, such that $\beta_{1}=0$ and $\beta_{2}=\beta_{3}=\beta_{4}=1$. In this case, homogeneous regions are reconstructed faster, but at the cost of smoothed structures.

Let us point out the fact that we chose to initialize the views to synthesize with black pixels. This choice is interesting to observe the diffusion model behaviour. However, it penalizes the computational time. Indeed, it takes a higher number of iterations to diffuse the color information, compared to the case where this initialization would be based on a combination of neighboring views. The combination of neighboring views would give initial views with blurry areas. Even if the model is able to structure them, this initialization may introduce slightly biased structures yielding local minima when running the diffusion. In our experiments, the best results have been 


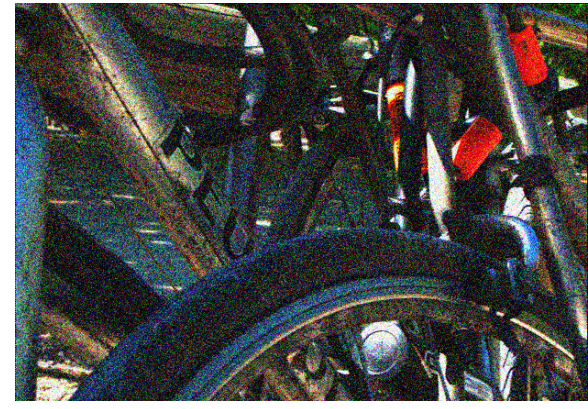

(a) Overall PSNR $14.15 \mathrm{~dB}$

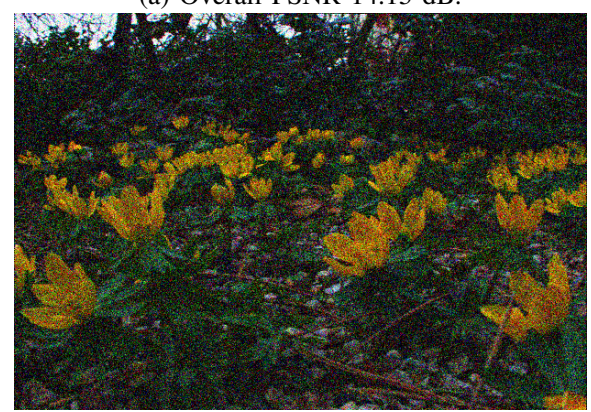

(d) Overall PSNR $14.15 \mathrm{~dB}$.

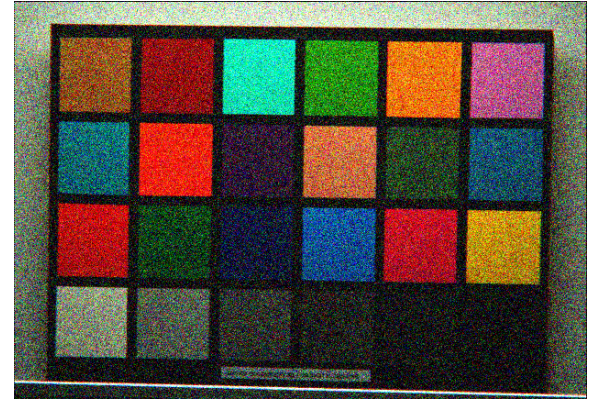

(g) Overall PSNR $14.15 \mathrm{~dB}$

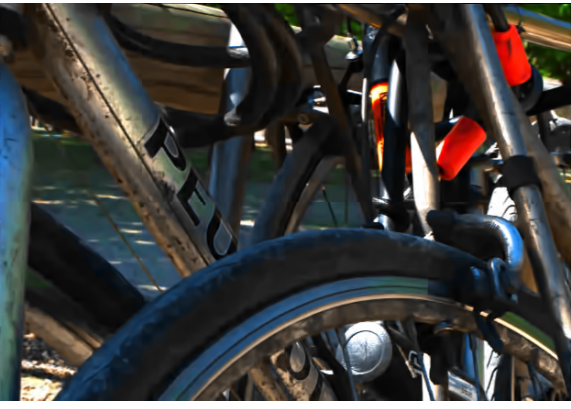

(b) Overall PSNR $29.91 \mathrm{~dB}$.

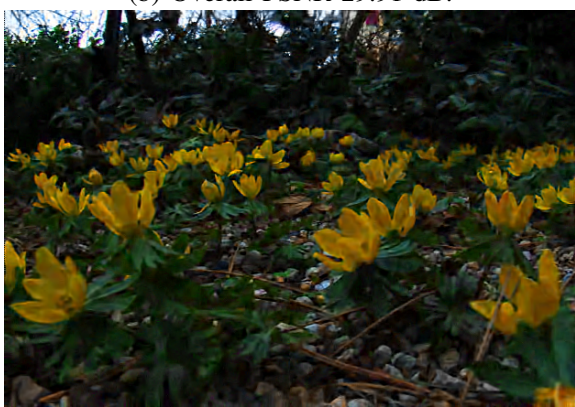

(e) Overall PSNR $28.47 \mathrm{~dB}$.

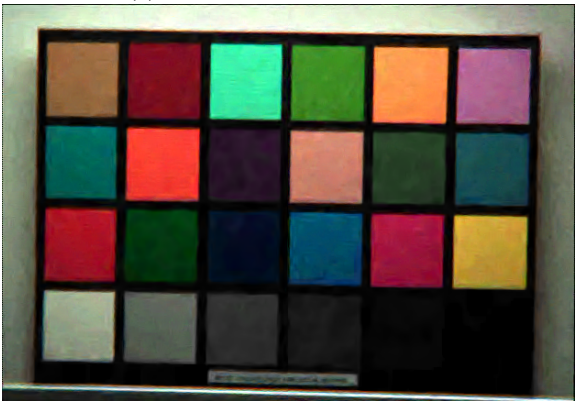

(h) Overall PSNR $30.22 \mathrm{~dB}$

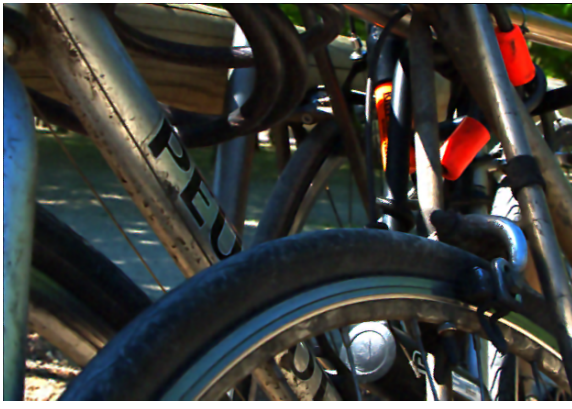

(c) Overall PSNR $30.10 \mathrm{~dB}, 19$ iterations.

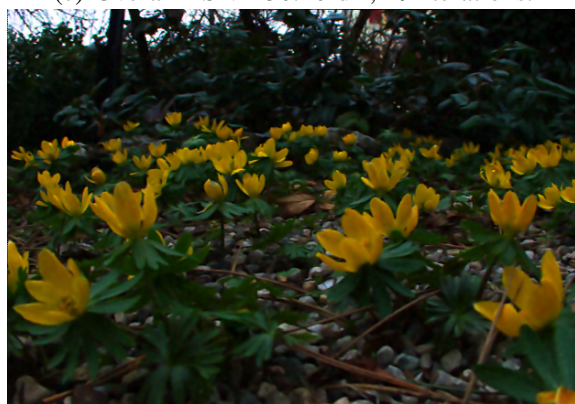

(f) Overall PSNR $30.54 \mathrm{~dB}, 17$ iterations.

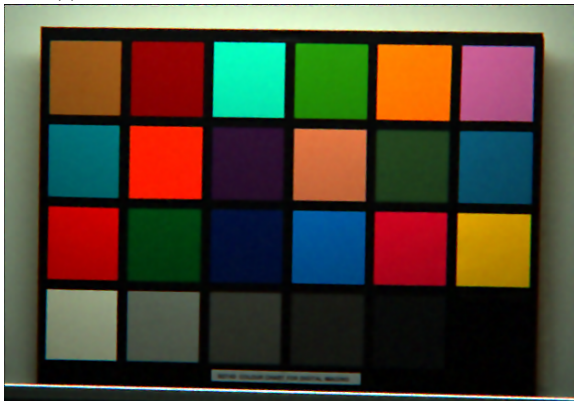

(i) Overall PSNR $30.82 \mathrm{~dB}, 23$ iterations.

Fig. 10: Denoising of the Bikes, Flowers and Color Chart 1 light fields [33]. Center views. (a,d,g) Gaussian noise of $\sigma=\frac{50}{255}$. (b,e,h) Result obtained using LFBM5D with parameterization provided by the authors [2]. (c,f,i) Using our method. An extended quantitative comparison with other methods can be found in Table I.

obtained using a black initialization.

\section{B. Spatial interpolation}

Tensor diffusion can also find applications in the field of spatial interpolation. 2D anisotropic diffusion has already been considered for image magnification. It is nevertheless interesting to show how $4 \mathrm{D}$ anisotropic diffusion can extend structures in the $4 \mathrm{D}$ ray space for light field spatial interpolation. For this purpose, we spatially downsize all light field views and then try to restore the resolution back to the original one, as explained in Fig. 20. The spatial interpolation process is initialized using nearest neighbor interpolation. Indeed, linear and cubic interpolation can introduce a bias with respect to real structures, due to their averaging effect. However, for even magnification factors, the nearest neighbor method can introduce a one pixel shift in the initialization. In the following experiments, the model parameters are set as $\beta_{1}=0$, $\beta_{2}=\beta_{3}=\beta_{4}=1$ and $w_{u}=w_{v}=0$. For a magnification factor 2, we set $w_{x}=w_{y}=0$, and for a factor 3, because it is necessary to help detecting structures orientation, we set $w_{x}=w_{y}=0.8$.
Tables III gives the PSNR obtained with the diffusion based light field spatial interpolation method with two magnification factors (2 and 3), in comparison with a nearest or a bicubic interpolation. The table gives the PSNR values both for the luminance only and for the three RGB color components. The light fields are from the EPFL, INRIA [35], and HCI [37] datasets. It is to be noted that our method yields lower PSNR than bicubic interpolation for a magnification factor of 2 for Cone Head, Monas Room and Watch light fields. The reason comes from the repetition of small structures in the scene which are sensitive to initial shifting of nearest neighbor initialization. On average, the anisotropic diffusion yields a $0.84 \mathrm{~dB}$ increment as compared to bicubic for factor 2 magnification, and a $1.87 \mathrm{~dB}$ increment for factor 3 . These numbers can be related to those obtained by state of the art methods applied to spatial super-resolution, despite the different experimental setups. That is, original low resolution pixels are preserved in our case, whereas spatial super-resolution methods produce blurring of low resolution images due to the downsampling method (floating point magnification factors). In [38], their method and the one proposed by [39] produce 


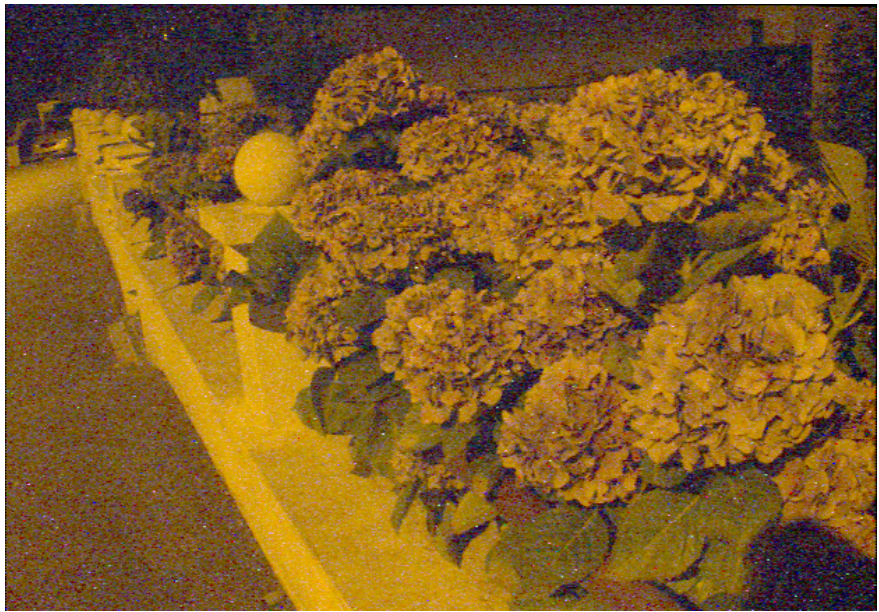

(a) Original center view.

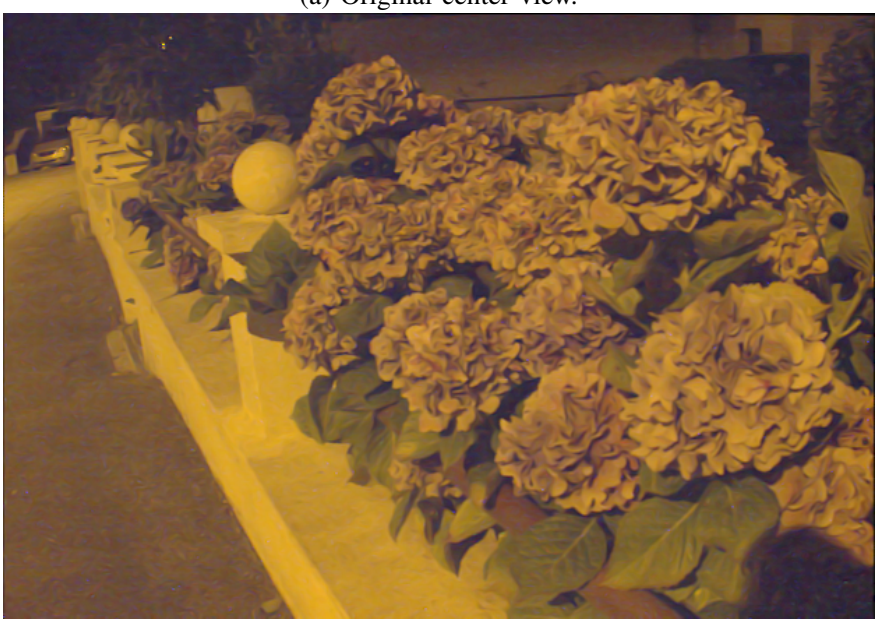

(b) After 15 iterations.

Fig. 11: Denoising of light field under low illumination conditions (LowLight_Flowers capture from the INRIA dataset [35]). The noise present in the input image results only from the poor capture conditions (no synthetic noise added). The denoising model is parameterized by $\rho_{4}=0.4, \rho_{3}=\frac{1}{2} \rho_{4}$, $\rho_{2}=\frac{1}{4} \rho_{3}$, and $\rho_{1}=\frac{1}{8} \rho_{2}$.

a $1.43 \mathrm{~dB}$ increase as compared to bicubic reconstruction for a magnification factor 3 . We can therefore see that spatial interpolation using anisotropic diffusion yields a coherent increase as compared to spatial super-resolution methods. Fig. 21 shows some visual results for the light field Papillon, with a magnification factor of 3 . The second row shows that the proposed method enables a better recovery of fine details (see e.g. legs of the butterfly). More visual comparisons can be found on the project web site ${ }^{2}$.

\section{Light field regularization and disparity estimate}

Disparity estimation methods based on local structure orientations in epipolar plane images [1] benefit from the ability to easily compute disparity everywhere on the light field. However, they suffer from inter-view inconsistencies and noise (even low) in homogeneous area. These drawbacks can yield noisy disparity estimate and poor confidence information $\phi$,

${ }^{2}$ http://clim.inria.fr/research/AD4D that we define empirically as a function of the quantity of spatio-angular structure in the $2 \mathrm{D}$ epipolar plane images: $\phi\left(\lambda_{+}, \lambda_{-}\right)=\left(\lambda_{+}+\lambda_{-}\right)^{0.2}$. One can refer to (1) for the two dimensional context. We propose to apply our method as a preprocessing of such disparity estimation methods. The goal is to enforce a maximum regularization of structures with minimum deformations. Using the denoising model introduced in Section IV, the diffusion has to operate only on the fourth eigenvector direction $\nu_{4}$. This parametrization is therefore the same as setting $\rho_{r}=\infty$ and $\alpha=\infty$. In Fig. 22a-b, we observe that the regularization of the light field strengthens the continuity of the confidence estimate. The application of a same lower bound threshold on confidence highlights how homogeneous areas without disparity information are more easily detected. In Fig. 22e-f, we can see that the preprocessing regularization yields a more robust disparity estimate. Of course, the regularization of structures could also be enforced using third or second order eigenvectors for the diffusion, but at the cost of a greater structure smoothing. Such trade-off has been discussed in Section IV.

\section{Inpainting}

Tensor diffusion has been used for reconstructing removed parts of 2D images, such as in [8]. The idea is to diffuse color information inside the masked area in the direction of structures present at edges of the mask. This approach let the information to fill in the inpainted area so that the continuity of structures is preserved. In Fig. 17, we show that our method is able fill the area of the three lines of text with coherent information regarding the neighboring structures.

\section{CONCLUSION}

Regularization of light fields in the 4D ray space provides an efficient method for a wide range of applications. Using structural properties in the four dimension space, we showed that structural oriented diffusion can drastically reduce noise while keeping most of the essential spatio-angular structures of the light field. The denoising model we proposed offers an easy parametrization and can be adapted according to the purpose (smoothness, structures sharpness, process speed). We believe that other models can be designed, to benefit even more of the angular consistency of noised views, and to tackle more efficiently the sparsity and its inherent 4D sharpness. We have also shown promising results for spatial and angular interpolation, as well as for inpainting. Indeed, by simply tuning the weights of the tensors obtained by each eigen component, it is possible to apply the method to a variety of inverse problems associated to light fields.

\section{REFERENCES}

[1] B. Goldluecke and S. Wanner, "The variational structure of disparity and regularization of 4D light fields," in IEEE Conf. on Computer Vision and Pattern Recognition (CVPR), June 2013, pp. 1003-1010.

[2] M. Alain and A. Smolic, "Light field denoising by sparse 5D transform domain collaborative filtering," in IEEE International Workshop on Multimedia Signal Processing, Oct. 2017.

[3] P. Allain, L. Guillo, and C. Guillemot, "Light field denoising using 4D anisotropic diffusion," in IEEE International Conf. on Acoustic, Speech and Signal Processing (ICASSP), May 2019. 


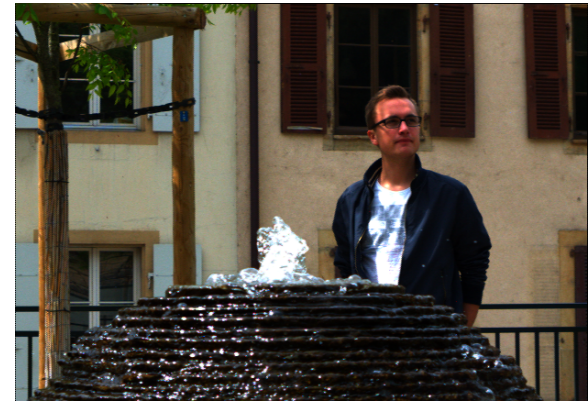

(a) Original center view.

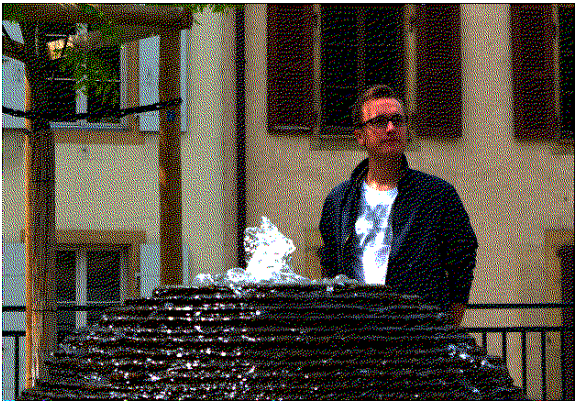

(b) Quantized on 3 bits, overall PSNR $8.42 \mathrm{~dB}$.

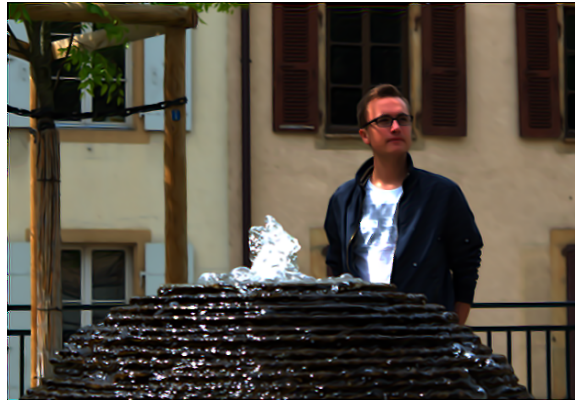

(c) Restored after 50 iterations, overall PSNR 27.27 dB.

Fig. 12: Restoration of light field damaged using the Floyd-Steinberg dithering method with only 2 levels per color channel (Fountain and Vincent 2 light field of the EPFL dataset [33]). The denoising model is parameterized by $\rho_{4}=0.4, \rho_{3}=\frac{1}{2} \rho_{4}, \rho_{2}=\frac{1}{4} \rho_{3}$, and $\rho_{1}=\frac{1}{8} \rho_{2}$.

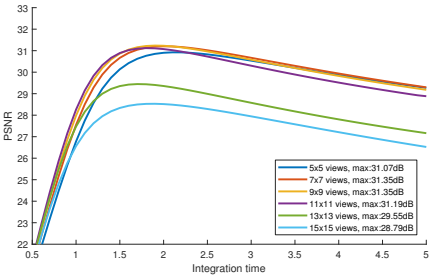

(a) Overall.

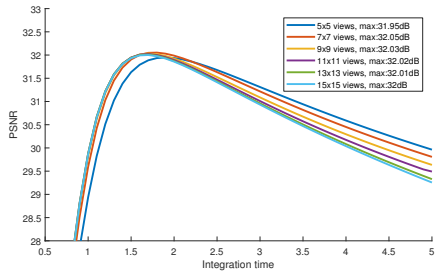

(b) Center view (rescaled plot).
Fig. 13: PSNR evolution when denoising the light fields with Gaussian noise $\left(\sigma=\frac{50}{255}\right)$. The PSNR values are averaged over the 12 test light fields of the EPFL dataset [33].

[4] P. Perona and J. Malik, "Scale space and edge detection using anisotropic diffusion," IEEE Trans. on Pattern Analysis and Machine Intelligence, vol. 12, pp. 629-639, 1990.

[5] J. Weickert, "Theoretical foundations of anisotropic diffusion in image processing," W. Kropatsch, R. Klette, F. Solina (Eds.), Theoretical foundations of computer vision, Computing Suppl. 11, Springer, Wien, p. 221-236, 1996

[6] S. Di Zenzo, "A note on the gradient of a multi-image," Comput. Vision, Graphics, Image Process., vol. 33, no. 1, pp. 116-125, Jan. 1986.

[7] J. Weickert, "Coherence-enhancing diffusion filtering," International Journal on Computer Vision, vol. 32, no. 2-3, pp. 111-127, 1989.

[8] D. Tschumperlé and R. Deriche, "Vector-valued image regularization with PDEs: A common framework for different applications," IEEE Trans. on Pattern Analysis and Machine Intelligence, vol. 27, no. 4, 2005.

[9] G. Sapiro and D. Ringah, "Anisotropic diffusion of multivalued images with applications to color filtering," IEEE Trans. Image Processing, vol. 5, no. 11, pp. 1582-1585, 19996.

[10] D. Tschumperlé and R. Deriche, "Constrained and unconstrained PDE's for vector image restoration," in Proc. 10th Scandinavian Conf. Image Analysis, 2001, pp. 153-160.

[11] D. Tschumperlé, "Fast Anisotropic Smoothing of Multi-Valued Images using Curvature-Preserving PDE's." International Journal of Computer Vision, vol. 68, no. 1, pp. 65-82, 2006.

[12] J. Pang and G. Cheung, "Graph laplacian regularization for image denoising: Analysis in the continuous domain," IEEE Trans. on Image Processing, vol. 26, no. 4, pp. 1770-1785, April 2017.

[13] K. Mitra and A. Veeraraghavan, "Light field denoising, light field superresolution and stereo camera based refocussing using a GMM light field patch prior," in Proc. CVPR Workshops, June 2012, pp. 22-28.

[14] A. Sepas-Moghaddam, P. L. Correia, and F. Pereia, "Light field denoising: exploiting the redundancy of an epipolar sequence representation," in Proc. 3DTV-Con, Jul. 2016.

[15] G. Boracchi, A. Foi, and K. Egiazarian, "Video denoising, deblocking, and enhancement through separable 4-D non local spatio-temporal transforms," IEEE Trans. on Image Processing, vol. 21, no. 9, pp. 3952 3966, 2012

[16] D. G. Dansereau, D. L. Bongiorno, O. Pizarro, and S. Williams, "Light field image denoising using a linear 4D frequency-hyperfan all- in-focus filter," in Proc. SPIE, vol. 8657, Feb. 2013.
[17] K. Dabov, A. Foi, and K. Egiazarian, "Image denoising by sparse 3-d transform-domain collaborative filtering," IEEE Trans. on Image Processing, vol. 16, no. 8, pp. 2080-2095, 2007.

[18] D. G. Dansereau, A. Eriksson, and J. Leitner, "Richardson-lucy deblurring for moving light field cameras," in IEEE Conf. on Computer Vision and Pattern Recognition (CVPR) workshop on Light Fields for Computer Vision, July 2017.

[19] A. Levin, W. Freeman, and F. Durand, "Understanding camera tradeoffs through a bayesian analysis of light field projections," in European Conf. on Computer Vision (ECCV), Oct. 2008.

[20] T. E. Bishop and P. Favaro, "The light field camera: Extended depth of field, aliasing, and superresolution," IEEE Trans. on Pattern Analysis and Machine Intelligence, vol. 34, no. 5, pp. 972-986, May 2012.

[21] R. A. Farrugia, C. Galea, and C. Guillemot, "Super resolution of light field images using linear subspace projection of patch-volumes," IEEE Journal of Selected Topics in Signal Processing, vol. 11, no. 7, pp. 1058-1071, Oct 2017.

[22] Y. Yoon, H.-G. Jeon, D. Yoo, J.-Y. Lee, and I. S. Kweon, "Learning a deep convolutional network for light-field image super-resolution," in IEEE International Conf. on Computer Vision and Pattern Recognition (CVPR), June 2015, pp. 57-65.

[23] S. Wanner and B. Goldluecke, "Variational light field analysis for disparity estimation and super-resolution," IEEE Trans. on Pattern Analysis and Machine Intelligence, vol. 36, no. 3, pp. 606-619, March 2014.

[24] T. Georgiev, K. C. Zheng, B. Curless, D. Salesin, S. Nayar, and C. Intwala, "Spatio-angular resolution tradeoff in integral photography," in In Eurographics Symposium on Rendering, 2006, pp. 263-272.

[25] S. Wanner and B. Goldluecke, "Variational light field analysis for disparity estimation and super-resolution," IEEE Trans. of Pattern analysis and machine intelligence, vol. 36, no. 3, 2013.

[26] S. Heber, R. Ranftl, and T. Pock, "Variational shape from light field," in EMMCVPR, ser. Lecture Notes in Computer Science, vol. 8081. Springer, 2013, pp. 66-79.

[27] Y. Yoon, H. Jeon, D. Yoo, J. Lee, and I. S. Kweon, "Learning a deep convolutional network for light-field image super-resolution," in IEEE International Conf. on Computer Vision Workshop (ICCVW), Dec 2015, pp. 57-65.

[28] J. Flynn, I. Neulander, J. Philbin, and N. Snavely, "Deep stereo: Learning to predict new views from the world's imagery," in 2016 IEEE Conference on Computer Vision and Pattern Recognition (CVPR), June 2016, pp. 5515-5524.

[29] N. K. Kalantari, T.-C. Wang, and R. Ramamoorthi, "Learning-based view synthesis for light field cameras," ACM Trans. on Graphics (Proceedings of SIGGRAPH Asia 2016), vol. 35, no. 6, 2016.

[30] S. Gortler, R. Grzeszczuk, R. Szeliski, and M. Cohen, "The lumigraph." 23 rd annual Conf. on Computer Graphics and Interactive Techniques, ACM, pp. 43-54, 1996.

[31] J. Weickert, "Coherence-enhancing diffusion of colour images," Image and Vision Computing, vol. 17, no. 3, pp. 201 - 212, 1999.

[32] D. Tschumperlé and R. Deriche, "Anisotropic Diffusion Partial Differential Equations in Multi-Channel Image Processing : Framework and Applications." in Advances in Imaging and Electron Physics (AIEP). Academic Press, 2007, pp. 145-209.

[33] M. Rerabek and T. Ebrahimi, "New light field image dataset," in Proc. QoMEX, 2016. 


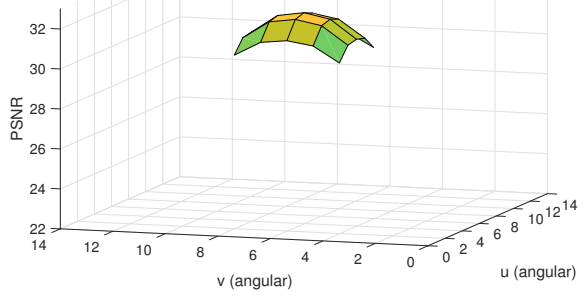

(a) $5 \times 5$ views

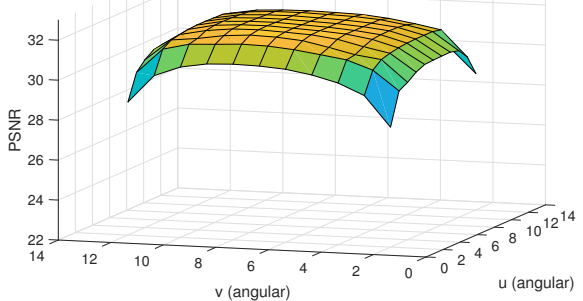

(d) $11 \times 11$ views

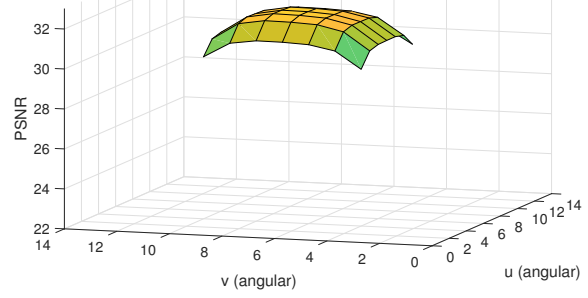

(b) $7 \times 7$ views

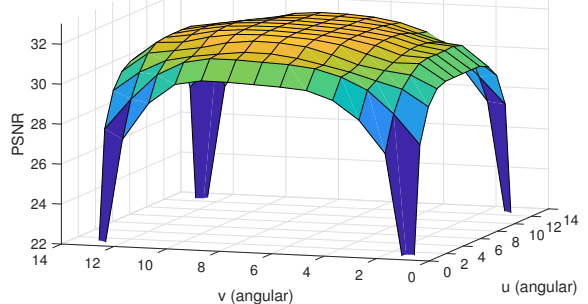

(e) $13 \times 13$ views

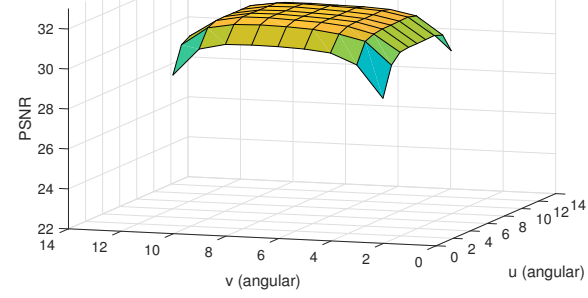

(c) 9x9 views

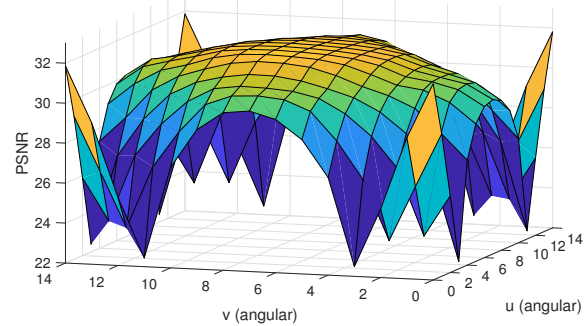

(f) $15 \times 15$ views

Fig. 14: Denoising PSNR values for each light field view, with different angular croppings, and after 20 iterations. Each PSNR value corresponds to the average over the 12 Lytro Illum light fields of the EPFL dataset [33]. The added noise is Gaussian and of standard deviation $\sigma=\frac{50}{255}$.

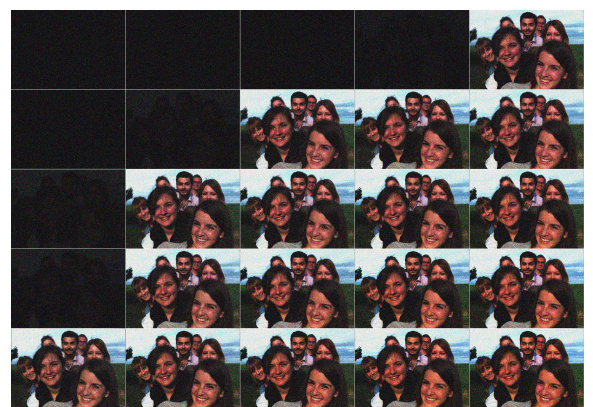

(a) Added Gaussian noise, $\sigma=\frac{50}{255}$.

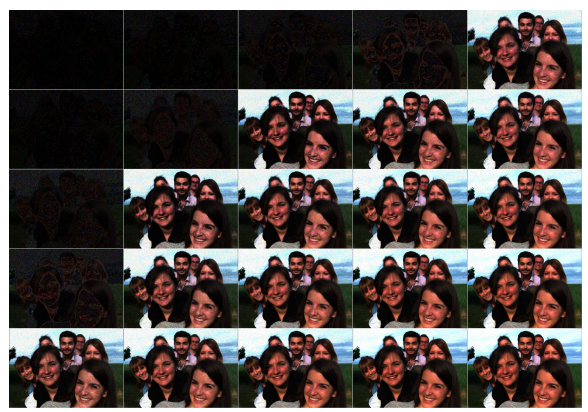

(b) Denoised, 20 iterations.

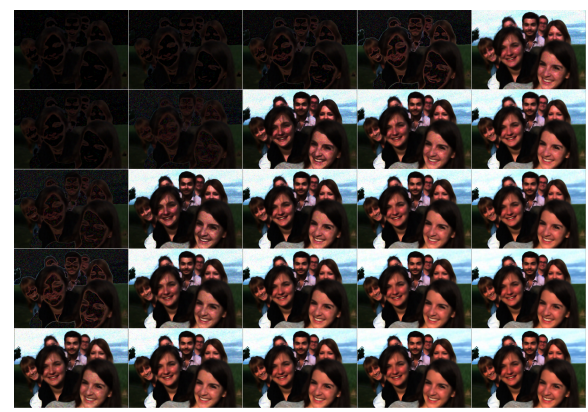

(c) Denoised, 50 iterations.

Fig. 15: Light field views of the Friends 1 light field of the EPFL dataset [33], cropped such that $u, v \in[0,4]$. During the denoising process, the diffusion tends to print views at the vignetted locations because of the aliasing.

[34] D. G. Dansereau, O. Pizarro, and S. B. Williams, "Decoding, calibration and rectification for lenselet-based plenoptic cameras," in 2013 IEEE Conf. on Computer Vision and Pattern Recognition, June 2013, pp. $1027-1034$.

[35] "Inria Lytro capture dataset," http://clim.inria.fr/DataSoftware.html.

[36] "The Stanford light field archive," http://lightfield.stanford.edu.

[37] "HCI light field dataset," http://lightfieldgroup.iwr.uni-heidelberg.de/.

[38] R. Farrugia and C. Guillemot, "A simple framework to leverage stateof-the-art single-image super-resolution methods to restore light fields," https://arxiv.org/abs/1809.10449, 2018.

[39] M. Rossi and P. Frossard, "Geometry-consistent light field superresolution via graph-based regularization," IEEE Trans. on Image Processing, vol. 27, no. 9, pp. 4207-4218, 2018.

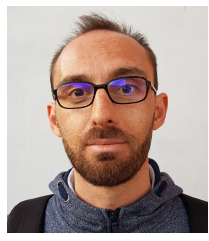

Pierre Allain received an engineering degree in 2007 at the Grenoble Institute of Technology. He received the $\mathrm{Ph} . \mathrm{D}$ degree in 2012 at the University of South Brittany. His research interests are human crowd simulation, modeling of biological systems, machine learning and image processing.

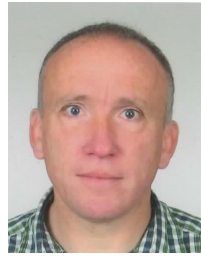

Laurent Guillo joined the CNRS and Irisa in 2002 as a research engineer after having spent 12 years in private companies mainly software publishers, in particular the world leader in business intelligence where he acted as technical leader and product manager. In the joint CNRS/Inria project team SIROCCO, he is in charge of software developments in the area of computational light field imaging.

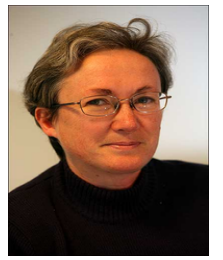

Christine Guillemot, IEEE fellow, is Director of Research at INRIA, head of a research team dealing with image and video modeling, processing, coding and communication. She holds a Ph.D. degree from ENST (Ecole Nationale Superieure des Telecommunications) Paris, and an Habilitation for Research Direction from the University of Rennes. From 1985 to Oct. 1997, she has been with FRANCE TELECOM, where she has been involved in various projects in the area of image and video coding. From Jan. 1990 to mid 1991, she has worked at Bellcore, NJ, USA, as a visiting scientist. Her research interests are signal and image processing, and in particular 2D and 3D image and video processing for various problems (compression, super-resolution, inpainting, classification). She has served as Associate Editor for IEEE Trans. on Image Processing (2000-2003, 20142016), for IEEE Trans. on Circuits and Systems for Video Technology (20042006), and for IEEE Trans. on Signal Processing (2007-2009). She has served as senior member of the editorial board of the IEEE journal on selected topics in signal processing (2013-2015) and is currently senior area editor of IEEE Trans. on Image Processing. 


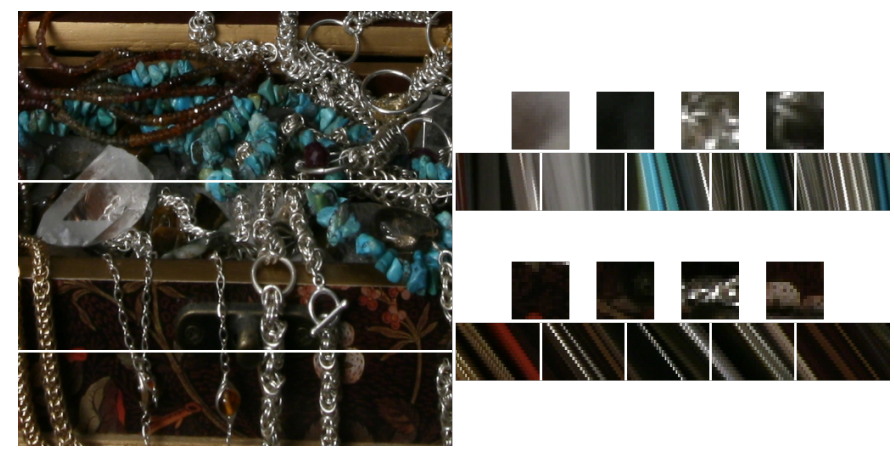

(a) Original. Disparity is lower than 1 at the top line location, and above 3 at the bottom line's.

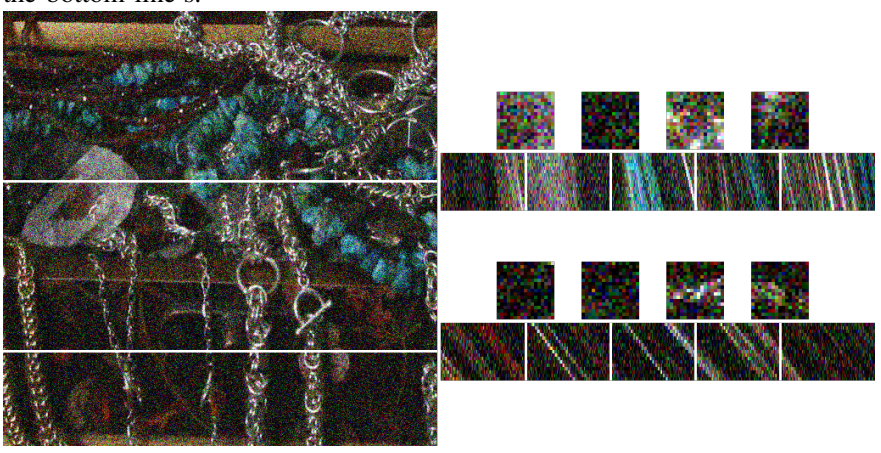

(b) Noised, overall PSNR $14.15 \mathrm{~dB}$

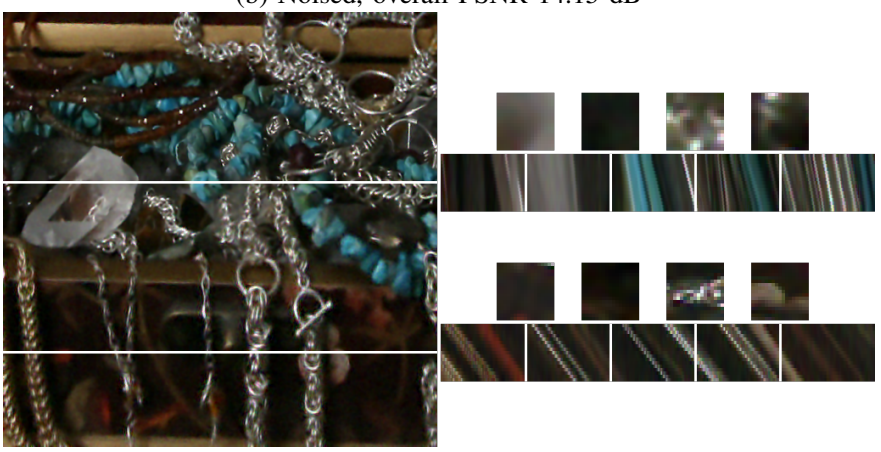

(c) Denoised, 15 iterations, overall PSNR $26.67 \mathrm{~dB}$

Fig. 16: Denoising results with the Treasure Chest light field (spatially cropped) of the Stanford dataset [36], with Gaussian noise $\left(\sigma=\frac{50}{255}\right)$. The model has difficulty coping with the high disparities at the foreground of the scene (bottom horizontal white line). Epipolar plane images are vertically stretched by a factor 4 for sake of clarity. Best viewed zoomed.

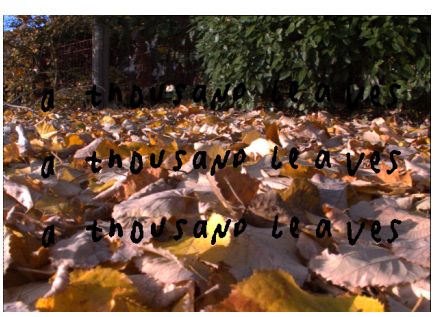

(a) Original. Center view

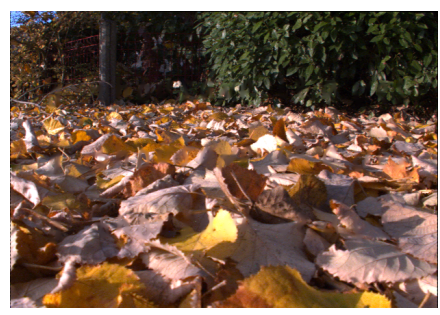

(b) Inpainted. Center view.

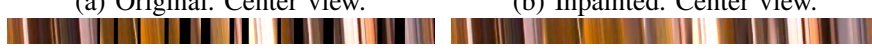

(c) Original. EPI at middle text.

(d) Inpainted. EPI at middle text.
(e) Original. EPI at bottom text.
(f) Inpainted. EPI at bottom text.

Fig. 17: Inpainting of the light field capture Leaves from the INRIA dataset. Experiment parameterized by: $\beta_{1}=0, \beta_{2}=\beta_{3}=\beta_{4}=1, \omega_{x}=\omega_{y}=$ $\omega_{u}=\omega_{v}=1.1$ and $w_{x}=w_{y}=2.9, w_{u}=w_{v}=0$. 


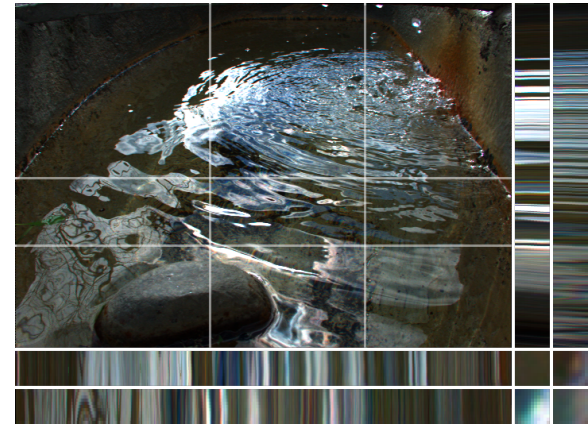

(a) Original.

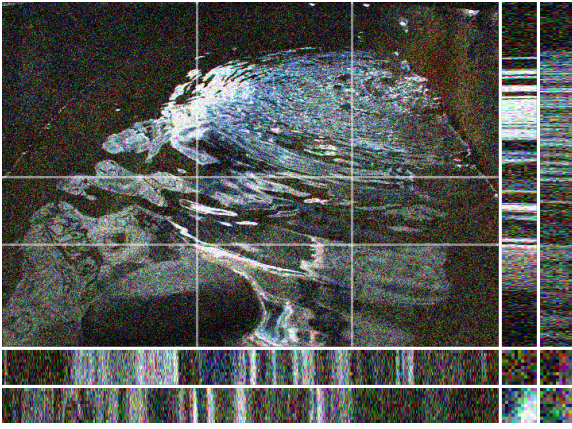

(b) Added Gaussian noise, $\sigma=\frac{50}{255}$. Overall PSNR 14.15 dB.

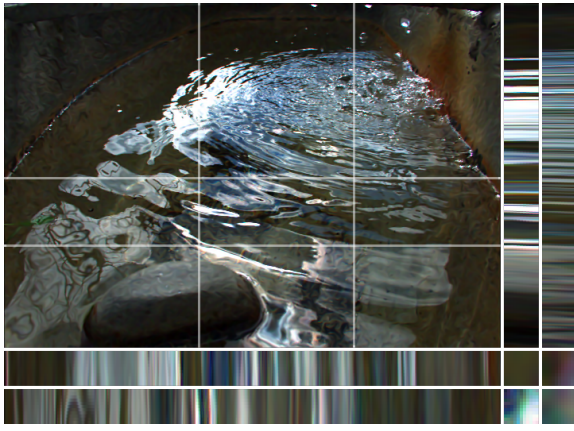

(c) Overall PSNR 30.28 dB, 30 iterations.

Fig. 18: Denoising results with the Fountain Pool light field [33], containing non-Lambertian surfaces. The denoising model is parameterized by $\rho_{4}=0.4$, $\rho_{3}=\frac{1}{2} \rho_{4}, \rho_{2}=\frac{1}{4} \rho_{3}$, and $\rho_{1}=\frac{1}{8} \rho_{2}$. Epipolar plane images are stretched in the angular direction by a factor 4 for sake of clarity. Best viewed zoomed.

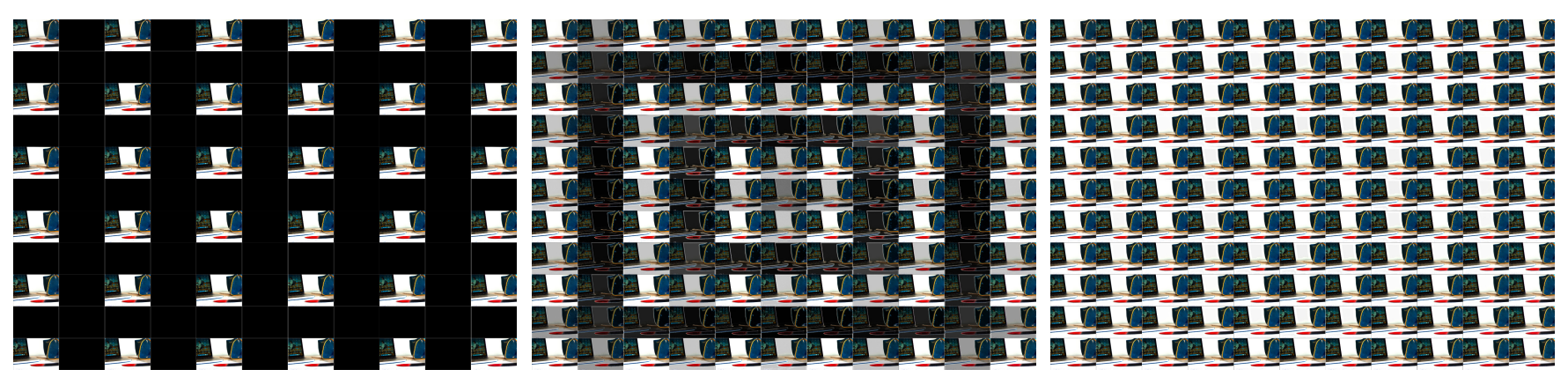

(a) Initial

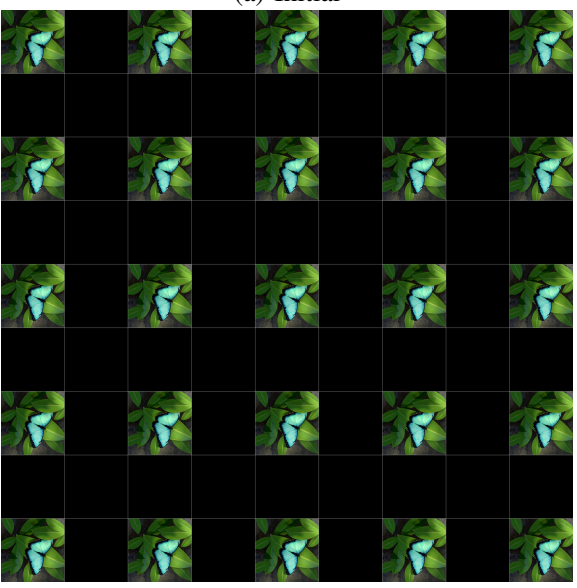

(d) Initial (b) 15 iterations

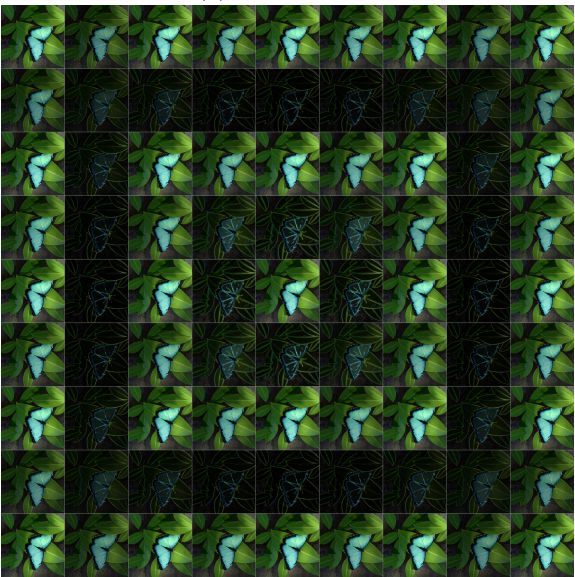

(e) 15 iterations (c) 200 iterations, PSNR $33.57 \mathrm{~dB}$

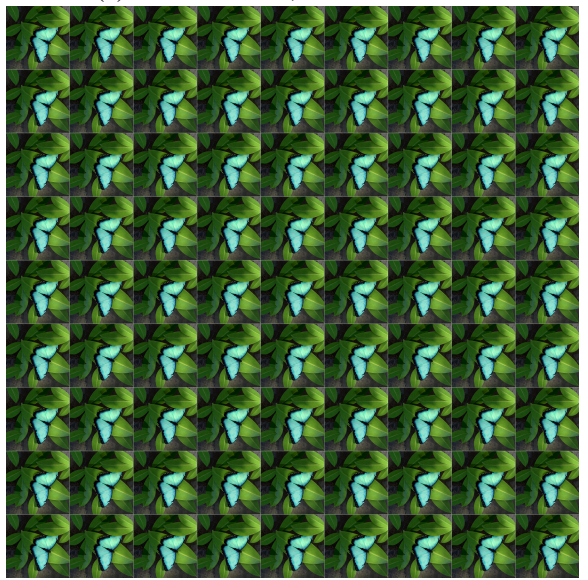

(f) 150 iterations, PSNR $41.82 \mathrm{~dB}$

Fig. 19: Angular interpolation for Desktop and Papillon light fields. The PSNR values are computed over all the synthesized views.

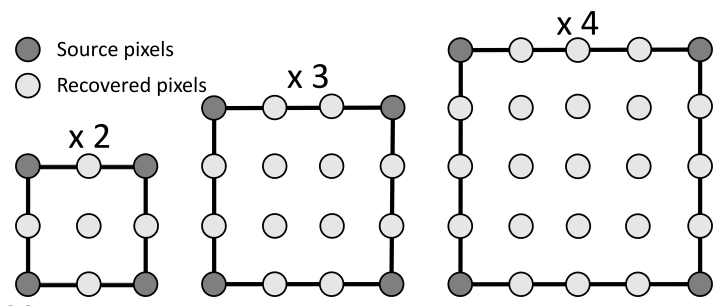

Fig. 20: Subsampling method for spatial interpolation. Only integer magnification factors are allowed. For performance evaluation, if image size does not match the partitionning, a cropping based on the corresponding modulo is applied. 


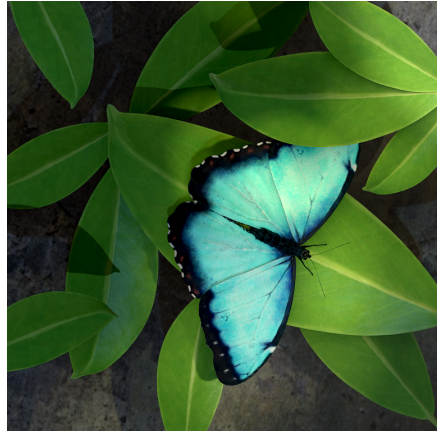

(a) Original

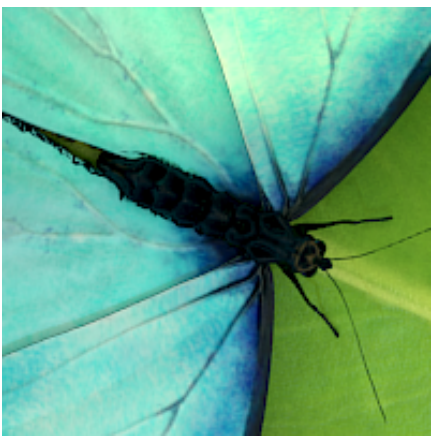

(e) Close-up of (a)

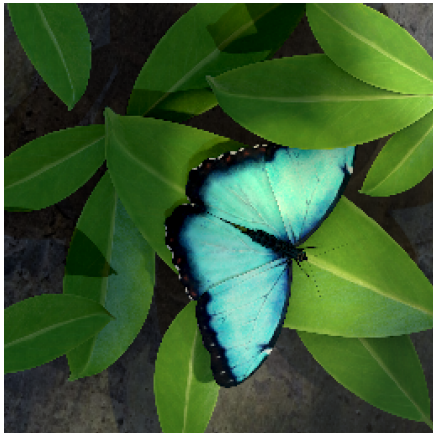

(b) Nearest neighbor interpolation PSNR $29.71 \mathrm{~dB}$

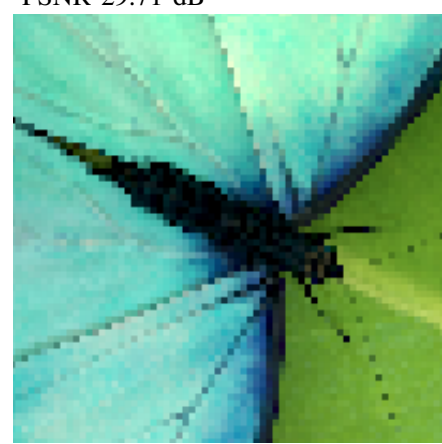

(f) Close-up of (b)

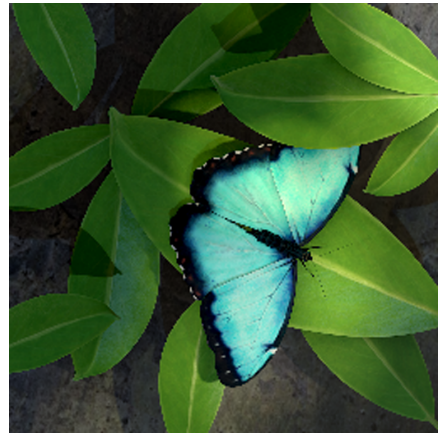

(c) Bicubic interpolation. PSNR 32.22 $\mathrm{dB}$

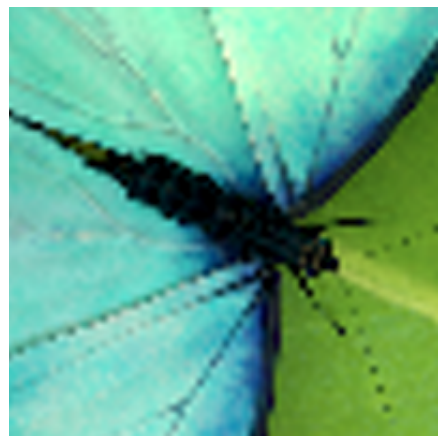

(g) Close-up of (c)

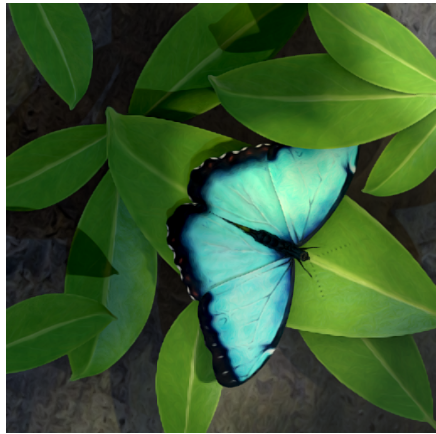

(d) Anisotropic tensor diffusion, 25 iterations. PSNR $34.92 \mathrm{~dB}$

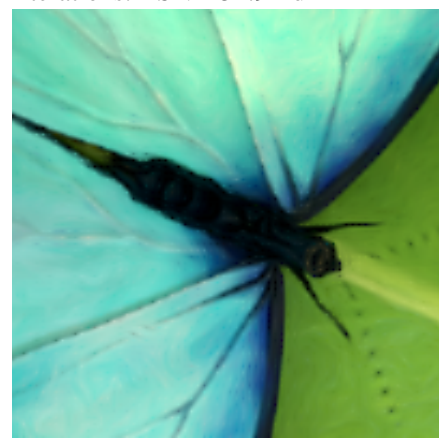

(h) Close-up of (d)

Fig. 21: Spatial interpolation example. Original light field (a,e) is spatially down sampled by a factor 3 and reconstructed to original dimensions (b-d and f-h). For this experiment, we set : $\omega_{x}=\omega_{y}=2.3$ and $\omega_{u}=\omega_{v}=1.1$, and $w_{x}=w_{y}=w_{u}=w_{v}=0$.

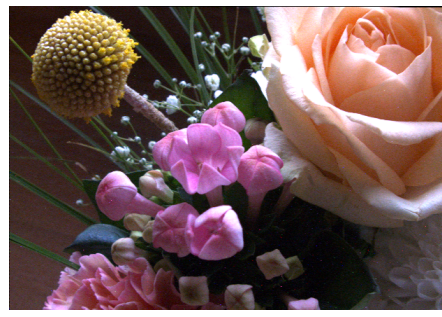

(a) Original (center view) and epipolar spatio-angular confidence.

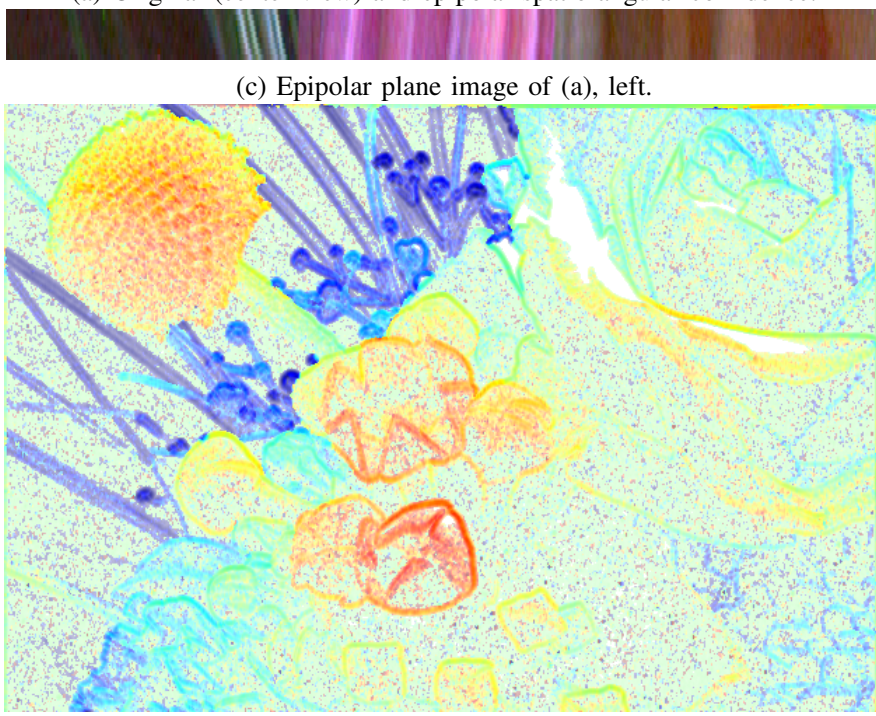

(e) Disparity estimate, opacity indexed to confidence.

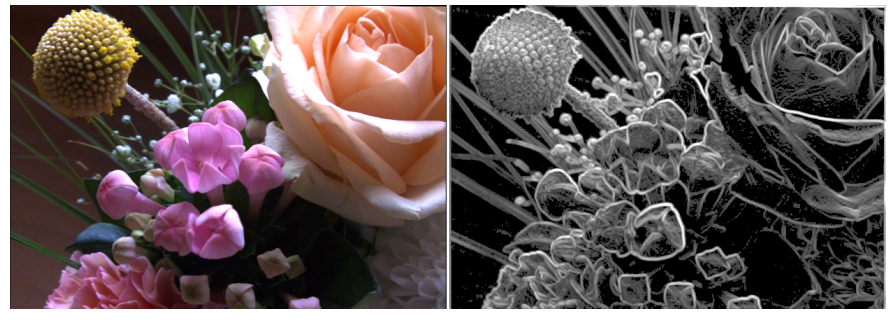

(b) Regularized (center view) and epipolar spatio-angular confidence.

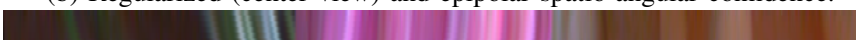

(d) Epipolar plane image of (b), left.

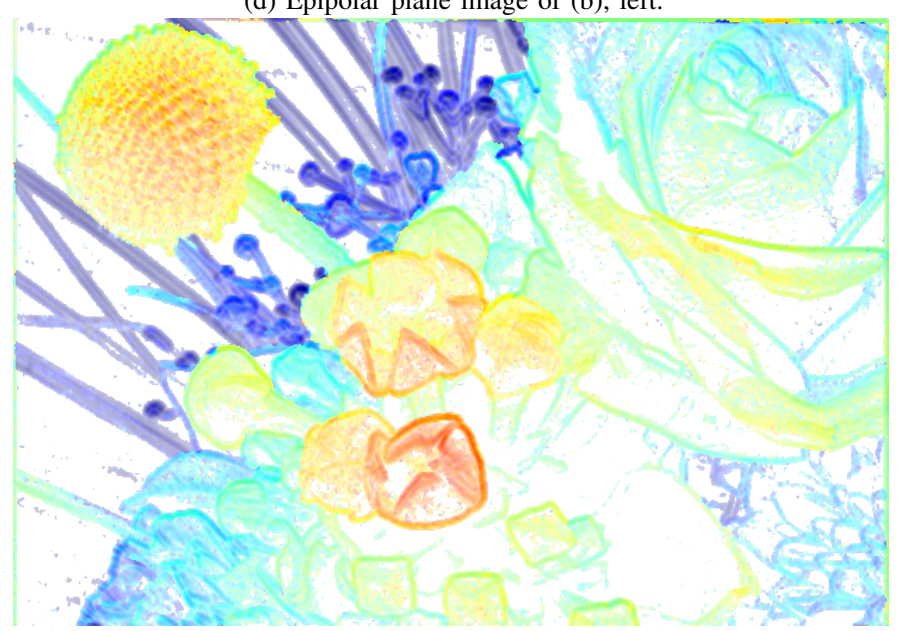

(f) Disparity estimate, opacity indexed to confidence.

Fig. 22: Light field regularization for disparity estimation enhancement. Diffusion is performed only in the fourth eigenvector $\nu_{4}$ direction, and yields a more robust epipolar disparity estimate. Left: using original light field. Right: using regularized light field. Epipolar spatio-angular confidence $\phi$ in (a) and (b) is thresholded identically to filter out low confidence value, and used as alpha channel for disparity display in (e) and (f). The light field is BouquetFlower2 from the INRIA dataset. 